\title{
Numerical methods for hydraulic fracture propagation: a review of recent trends ${ }^{* \dagger}$
}

\author{
Brice Lecampion ${ }^{\ddagger 1}$, Andrew Bunger ${ }^{2,3}$, and Xi Zhang ${ }^{4}$ \\ ${ }^{1}$ Geo-Energy Lab - Gaznat chair on Geo-Energy, Swiss Federal Institute of Technology in \\ Lausanne, EPFL-ENAC-IIC-GEL, Station 18, Lausanne CH-1015, Switzerland \\ ${ }^{2}$ Department of Civil and Environmental Engineering, University of Pittsburgh, PA, USA \\ ${ }^{3}$ Department of Chemical and Petroleum Engineering, University of Pittsburgh, PA, USA \\ ${ }^{4}$ CSIRO Energy, Clayton, Vic, Australia
}

November 14, 2017

\section{Highlights}

- A review of recent numerical methods for the modeling of hydraulic fracture propagation.

- Proper validations against semi-analytical solutions are essential and are often lacking.

- The drive toward more model complexity does not always lead to better predictability.

*Invited review - Journal of Natural Gas Science \& Engineering,submitted July 28, 2017, accepted Oct. 28 2017, DOI: https://doi.org/10.1016/j.jngse.2017.10.012

$\dagger$ 'Author's manuscript, (C)2017. This manuscript version is made available under the CC-BYNC-ND 4.0 license http://creativecommons.org/licenses/by-nc-nd/4.0/

${ }^{\ddagger}$ Corresponding author: brice.lecampion@epfl.ch 


\section{Contents}

1 Introduction $\quad 4$

2 Problem Formulation $\quad 6$

2.1 The basic hydraulic fracturing model . . . . . . . . . . . . 7

2.1.1 Linear elasticity . . . . . . . . . . . . . . . 7

2.1.2 Linear elastic fracture mechanics . . . . . . . . . . . . . 9

2.1.3 Fluid flow in the fracture . . . . . . . . . . . . . . . . 11

2.1.4 Carter's leak-off . . . . . . . . . . . . . . 13

2.1.5 Boundary conditions . . . . . . . . . . . . . . 15

2.1.6 Multiscale tip asymptotics . . . . . . . . . . . . . . 16

2.2 Model Extensions . . . . . . . . . . . . . . . . . . . . . 17

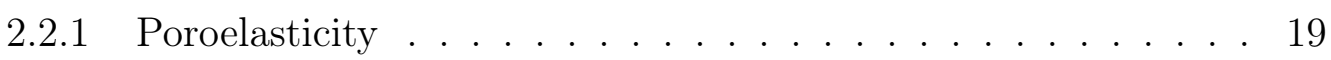

2.2.2 Fluid non-linearities . . . . . . . . . . . . . . . . . . . . . 19

2.2.3 Solid non-linearities . . . . . . . . . . . . . . . . . 20

2.2.4 Non-linear injection conditions . . . . . . . . . . . . 21

3 The challenges of a numerical solution and the different schemes 21

3.1 Solution of the elasto-hydrodynamics system . . . . . . . . . . . 22

3.2 Fracture front tracking . . . . . . . . . . . . . . . 23

4 Advances in algorithms for continuum models $\quad 24$

4.1 Schemes using the multi-scale hydraulic fracture tip asymptotes for fracture front tracking . . . . . . . . . . . . . . . . 25

4.2 Boundary element schemes . . . . . . . . . . . . . . . . . 26

4.3 Finite element schemes . . . . . . . . . . . . . . . . . . 27

4.3.1 Fracture growth with re-meshing . . . . . . . . . . . . 27

4.3.2 Growth along a pre-defined path . . . . . . . . . . . 28

4.3.3 Extended / Generalized finite element formulations . . . . . . 29

4.3.4 Phase field methods . . . . . . . . . . . . . . . . . . . 32

4.4 Meshless methods . . . . . . . . . . . . . . . . . 35

4.4.1 Element free galerkin . . . . . . . . . . . . . . 35

4.4.2 Smooth Particle Hydrodynamics . . . . . . . . . . . . . . 36

5 Meso-scale models $\quad 36$

$\begin{array}{lll}6 & \text { Engineering models } & 37\end{array}$ 
$\begin{array}{lll}7 & \text { The necessity of verification and validation } & 40\end{array}$

8 Conclusions 44 


\begin{abstract}
Development of numerical methods for hydraulic fracture simulation has accelerated in the past two decades. Recent advances in hydraulic fracture modeling and simulation are driven by increased industry and research activity in oil and gas, a drive toward consideration of more complex behaviors associated with layered and naturally-fractured rock formations, and a deepening understanding of the underlying mathematical model and its intrinsic challenges. Here we review the basic approaches being employed. Some of these comprise enhancements of classical methods, while others are imported from other fields of mechanics but are completely new in their application to hydraulic fracturing. After a description of the intrinsic challenges associated with the mechanics of fluid-driven fractures, we discuss both continuum and meso-scales numerical methods as well as engineering models which typically make use of additional assumptions to reduce computational cost. We pay particular attention to the verification and validation of numerical models, which is increasingly enabled by an ever-expanding library of laboratory experiments and analytical solutions for simple geometries in a number of different propagation regimes. A number of challenges remain and are amplified with a drive toward fully-coupled, three-dimensional hydraulic fracture modeling that accounts for host-rock heterogeneity. In the context of such a drive to complex models, we argue that the importance of best-practice development that includes careful verification and validation is vital to ensure progress is constrained by the appropriate underlying physics and mathematics with a constant attention to identifying conditions under which simpler models suffice for the intended modeling purposes.
\end{abstract}

\title{
1 Introduction
}

Mathematical modeling for the prediction of hydraulic fracture propagation is nearly as old as hydraulic fracturing technology itself. The first successes demonstrating hydraulic fracturing for oil and gas wells were obtained in the late 1940s and early 1950s (Montgomery and Smith, 2010), and by the mid 1950s tens of thousands of wells were stimulated (Economides and Nolte, 2000, Ch. 5A). Hydraulic fracture modeling followed closely behind, with seminal works such as Khristianovic and Zheltov (1955), Howard and Fast (1957), and Perkins and Kern (1961), with models laid out in these early works being revisited and refined by Geertsma and De Klerk (1969) and Nordgren (1972). By the time of the first hydraulic fracturing review paper (Hassebroek and Waters, 1964), the basic influences of fluid viscosity, injec- 
tion rate, and host rock permeability were recognized, albeit still in a somewhat qualitative sense.

Over the decades that followed, hydraulic fracture simulation became an integral part of design and analysis of treatments both for stimulation and reservoir characterization. Some major accomplishments include height growth prediction (after Simonson et al., 1978), tip-screenout design for proppant scheduling (after Nolte, 1986), and analysis of pressure decline following hydraulic fracture shut-in for reservoir characterization purposes (after Nolte and Smith, 1981).

Following the boom in hydraulic fracturing associated with the development of unconventional reservoirs since the mid 2000s, the number of technical contributions on this topic has drastically increased. In addition to an increase in activity, the nature of the hydraulic fractures used for stimulation of unconventional reservoirs has driven the development of increasingly complex models that are able to account for interaction between hydraulic fractures and pre-existing fracture networks, simultaneous growth of multiple hydraulic fractures, 3D fracture growth in the vicinity of the wellbore, and so forth.

All of this has taken place with concurrent developments that more clearly recognize the consequences of the fact that propagation of hydraulic fractures in rock is, by nature, a moving-boundary problem (the fracture geometry evolves with time). In its simplest form it couples the elastic deformation of the rock (a non-local equation) with lubrication flow in the fracture as well as fracture propagation condition. It is now well understood that hydraulic fracture propagation is directly influenced by the competition of two dissipative processes (viscous flow and fracture surface creation) as well as the relative amount of fluid leak-off in the rock matrix in comparison to the amount of fluid stored in the fracture during propagation (Detournay, 2016). The propagation behavior thus widely differs depending on the dominant effects resulting in different propagation "regimes" depending on the problem parameters (viscosity/storage dominated, toughness/leak-off dominated, and so forth). The competition between these physical processes intrinsically amplifies near the tip of the fracture resulting in a multi-scale structure of the solution which is now well established theoretically (Garagash et al., 2011) and confirmed by laboratory experiments (Bunger and Detournay, 2008; Xing et al., 2017). As a result of this multi-scale structure, the estimate of the propagation velocity of hydraulic fracture at a given time poses tremendous challenges to any numerical models even in the simplest case of a planar fracture geometry.

Recent advances in hydraulic fracture modeling and simulation occur, then, at the confluence of drivers that include increased activity, a drive toward expanded complexity, and a deepening understanding of the underlying mathematical model 
and its intrinsic challenges. In this paper, we present an overview of some of these recent advances. This work follows in the steps of previous reviews (Mendelsohn, 1984a,b; Adachi et al., 2007), providing a needed update in light of the past decade of progress. Our focus is specifically on numerical methods, not on the application of numerical modeling to a particular engineering problem or a specific field case. We restrict our review to the propagation of hydraulic fractures and will not address other important topics in the context of reservoir stimulation such as proppant transport, fracture clean-up, production of hydraulically fractured reservoir, etc. In an engineering sense, we focus here on the so-called fracture placement problem. Our aim is to try to clarify the advantages and drawbacks of some different numerical techniques in order to help in choosing the most adequate method for a specific problem. We refer the reader to Economides and Nolte (2000); Smith and Montgomery (2015) for a thorough description of the different engineering aspects of a hydraulic fracturing treatment for the stimulation of oil and gas wells.

Before reviewing the benefits and limitations of the recently developed continuum and meso-scale numerical methods, we first briefly review the basic of the hydraulic fracture problem and its intrinsic peculiarities. We then discuss the advances of engineering-type models which typically perform additional physical hypothesis in order to speed up the numerical solution of the initial problem. We strongly argue for the required verification and validation of numerical models against correct analytical solutions and laboratory experiments. Finally, we discuss the importance of such verification and the benefits associated with being able to quantitatively compared different numerical options.

\section{Problem Formulation}

Hydraulic fractures predominantly propagate in mode I (opening mode) under the action of a fluid whose pressure must overcome the in-situ compressive stress field $\sigma_{i j}^{o}$ encountered at depth. Hence it is clear that the energy required to create new fracture surfaces must be accounted for in order to track the growth of a hydraulic fracture. This is typically done using the well-established concepts of fracture mechanics (e.g. Kanninen and Popelar, 1985). The growth of a hydraulic fracture differs, however, substantially from most other fracture mechanics problems. The fracture is loaded internally by a fluid whose pressure is varying in time and space along the fracture and is part of the solution of the problem. The elasticity problem is thus intrinsically coupled to the flow of fluid inside the fracture. This coupling is extremely stiff as the resistance to viscous fluid flow depends non-linearly on the 
fracture width. Another important aspect of the propagation of a fracture driven by an injected fluid is that it is inherently a volume controlled process. At any given time, the total injected volume $\mathcal{V}_{i n j}$ since the beginning of pumping is equal to the current fracture volume $\mathcal{V}_{\text {frac }}$ plus the volume of fluid "leaked-off" $\mathcal{V}_{\text {leak-off }}$ from the fracture faces onto the surrounding rock. Similarly to a test performed under displacement control in classical fracture mechanics, the fracture growth is thus stable (i.e. the pressure decreases in response to continuous injection and fracture growth).

In order to frame the discussion of the different numerical methods discussed in this paper, we briefly recall below the formulation of the hydraulic fracture propagation model (see also Detournay and Peirce (2014); Detournay (2016)) as well as some of its particularities and typical extensions.

\subsection{The basic hydraulic fracturing model}

For clarity of presentation, we consider here the case of a hydraulic fracture propagating in an infinite linear elastic isotropic medium (see Fig. 1) having an initial compressive stress field $\sigma_{i j}^{o}$.

\subsubsection{Linear elasticity}

Under quasi-static conditions encountered during hydraulic fracturing (recall that fracture growth is stable because it is volume controlled), the linear balance of momentum reduces to

$$
\partial_{x_{j}}\left(\sigma_{i j}-\sigma_{i j}^{o}\right)=0
$$

Here we use the convention of summation on repeated indices and write partial derivatives along $x_{j}$ as $\partial_{x_{j}}$. Tractions $t_{i}=\sigma_{i j} n_{j}$ are continuous across the fracture surfaces $S^{+}$and $S^{-}$, viz.

$$
t_{i}^{+}+t_{i}^{-}=0
$$

That is to say, the shear stresses transmitted from the fluid to the medium on the fracture face are negligibly small compared to the fluid pressure (a classical hypothesis also done in poroelasticity). The fluid pressure field $p$ acts on the normal to the fracture face

$$
t_{i}^{-} n_{i}^{-}=-p
$$




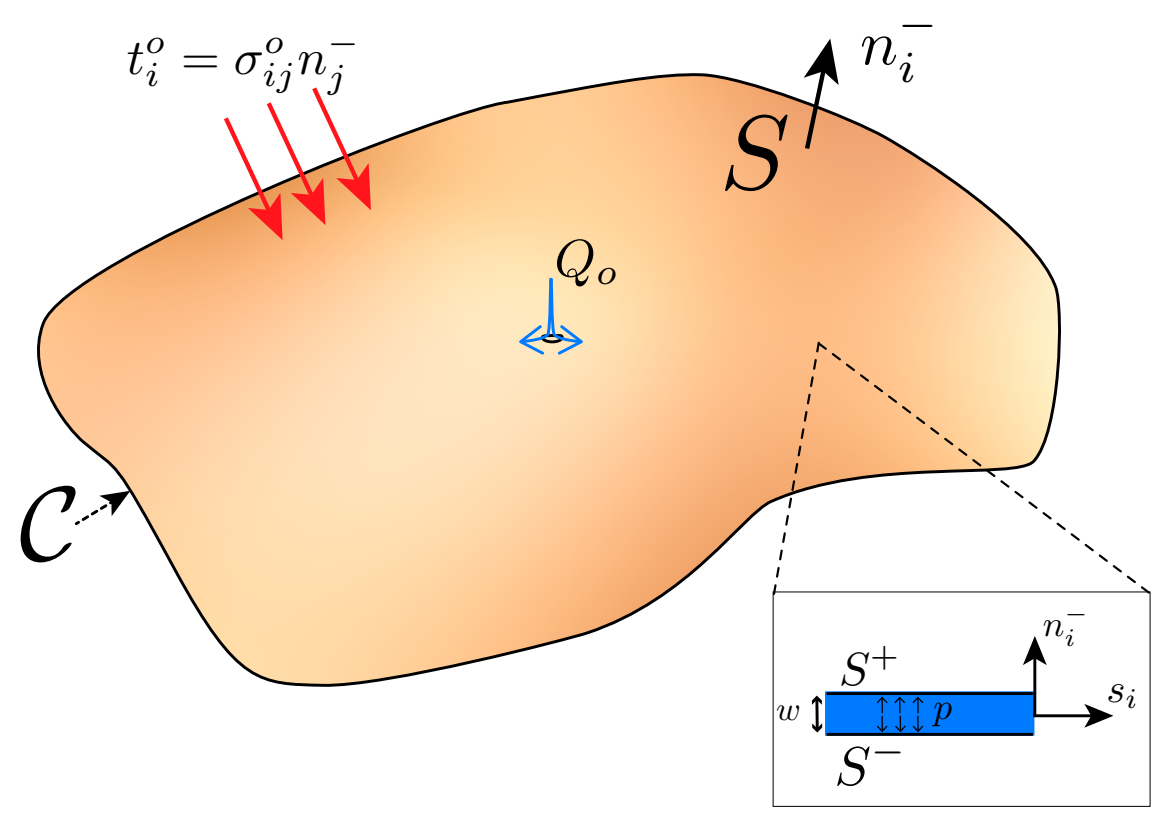

Figure 1: Sketch of a hydraulic fracture with surface $S$ and normal $n_{i}$ propagating in a pre-existing in-situ stress field $\sigma_{i j}^{o}$ due to fluid injection at a rate $Q_{o}$. Here, the contour $\mathcal{C}$ is the fracture front. The fracture opening $w$, fluid pressure $p$ and local coordinate system at a point is depicted in the inset. 
Assuming small strain linear elasticity, the relations between the stresses $\left(\sigma_{i j}\right)$ and strains $\left(\epsilon_{i j}\right)$ reduces to

$$
\epsilon_{i j}=\frac{1}{2}\left(\partial_{x_{j}} u_{i}+\partial_{x_{i}} u_{j}\right)=\frac{1+\nu}{E}\left(\sigma_{i j}-\sigma_{i j}^{o}\right)-\frac{\nu}{E}\left(\sigma_{k k}-\sigma_{k k}^{o}\right) \delta_{i j}
$$

Because of the presence of the crack, the displacement field $u_{i}$ exhibits a jump across the surfaces

$$
\llbracket u_{i} \rrbracket=u_{i}^{+}-u_{i}^{-}
$$

In particular, we write the fracture width (the opening component) as $w=\left(u_{i}^{+}-u_{i}^{-}\right) n_{i}^{-}$ (see Fig. 1). Note that a non-negativity constraint on the fracture opening applies in order to prevent any physical interpenetration $w \geq 0$. This linear elastic problem is closed by the condition of negligible displacement at infinity. The generalization to a finite body with any proper boundary conditions is straightforward.

The elastic problem described by eqs. (1)-(4) with proper boundary conditions can be solved by any type of numerical method (e.g. finite element). Among those, the boundary element method is particularly popular for fracture problems in homogeneous (or piece-wise homogeneous) medium due to its domain reduction, accuracy and ease of meshing. Using classical integral representations (Mogilevskaya, 2014, e.g.), the elastic problem can be schematically re-written as the following non-local operator:

$$
t_{i}^{-}-t_{i}^{o-}=\mathcal{L}_{i j}\left(\llbracket u_{j} \rrbracket\right)
$$

where $t_{i}^{o-}=\sigma_{i j}^{o} n_{j}^{-}$are the tractions on the fracture surfaces due to the initial compressive stress field. The integral operator $\mathcal{L}_{i j}$ is non-local and hypersingular (see Mogilevskaya (2014); Bonnet (1999); Hills et al. (1996) for more details on elastic fracture problems and their integral representations). Its numerical discretization results in a fully populated matrix. The previous representation of the elastic relation between displacement discontinuity over the fracture surface and the fracture net loading can also be obtained numerically after discretization by other numerical methods - using a numerical Green's function approach.

It is worth re-emphasing that both the fluid pressure field $p$ and the displacement jump \} u _ { i } \rrbracket \text { are unknowns. Their solution results from the coupling between elastic } deformation and fluid flow.

\subsubsection{Linear elastic fracture mechanics}

The driving force for fracture growth is the energy release rate $G$, which is equal to minus the derivative of the elastic potential energy with respect to an infinitesimal 
fracture surface $S$ increment under a constant load

$$
G=-\frac{\partial P}{\partial S}
$$

where the elastic potential energy is $P=\frac{1}{2} \int_{\Omega} \sigma_{i j} \epsilon_{i j} \mathrm{~d} V-\int_{\partial \Omega} \sigma_{i j} n_{j} u_{i} \mathrm{~d} S$ (see Rice and Drucker (1967); Rice (1968) for more details). More precisely, the linear elastic fracture energy criteria for fracture growth can be written as:

$$
\left\{\begin{array}{l}
\left(G-G_{c}\right) \mathrm{d} S=0 \\
G_{c} \mathrm{~d} S \geqslant 0
\end{array}\right.
$$

where the critical fracture energy release rate $G_{c}$ is a positive material constant. The previous criteria ensures that if $G<G_{c}$, no fracture growth occurs $(\mathrm{d} S=0)$, and $G=G_{c}$ during fracture growth $\left.(\mathrm{d} S>0)\right)$ which corresponds to a hypothesis of quasi-static growth equilibrium.

For a pure mode I hydraulic fracture growing in a plane perpendicular to the minimum in-situ stress, the previous energy growth criterion can be replaced by the equality of the mode I stress intensity factor $K_{I}$ with the material toughness $K_{I c}$. For such a fracture configuration, the mode I stress intensity factor $K_{I}$ is related to $G$ via Irwin's relation: $G=\frac{K_{I}^{2}}{E^{\prime}}$. The linear elastic fracture mechanics tip asymptotic for the fracture width $w$ as function of the distance to the fracture front $\hat{x}$ )

$$
w=\sqrt{\frac{32}{\pi}} \frac{K_{I}\left(1-\nu^{2}\right)}{E} \hat{x}^{1 / 2} \quad \hat{x} \ll 1
$$

can notably be used to obtain the stress intensity factor $K_{I}$.

For a pure opening fracture (i.e. a planar fracture), the quasi-static growth criteria reduce to $K_{I}=K_{I c}$ at all points locally along the fracture front $\mathcal{C}$. In the case of mixed-mode loading, it is well-established that the fracture will re-orient itself in order to maintain condition of pure opening mode at the fracture front Hutchinson and Suo (1991). Under mixed mode I \& II (opening and shearing) loading, different criteria to estimate the change of direction of fracture propagation have been proposed. The principle of local symmetry (i.e. zero mode II stress intensity factor locally), or the maximum tensile stress criteria (Erdogan and Sih, 1963) are the most commonly used. They both give relatively close estimate and agree with experimental results.

The case where all three modes of fracture propagation (opening mode, shear and anti-plane shear) occurs simultaneously along the fracture front remains challenging. 
The combination of opening and anti-plane shear modes results in the splitting of the fracture front (Cooke and Pollard, 1996). No general criteria to handle such a case is yet recognized as fully satisfactory although a number of recent advances have been made (Lin et al., 2010; Lazarus, 2011; Pham and Ravi-Chandar, 2016; Leblond et al., 2015; Chen et al., 2015) on that particular problem by the mechanics community.

\subsubsection{Fluid flow in the fracture}

The hypothesis of lubrication flow holds in a fracture which is intrinsically a long thin channel. The equations of thin-film lubrication are obtained from the width averaging (in the direction normal to the fracture wall) of the fluid mass and momentum conservation (see e.g. Szeri (2010)). The width averaged mass conservation for a fluid of density $\rho$ is given by:

$$
\partial_{t}(\rho w)+\partial_{s_{i}}\left(\rho w V_{i}\right)+2 \rho v_{L}=\rho Q_{i n j}(t) \delta\left(x-x_{i n j}\right)
$$

where $\delta$ is the Kronecker delta, $x_{i n j}$ the injection point, $V_{i}$ is the width averaged fluid velocity in the direction $s_{i}$ (in the fracture plane), and $v_{L}$ is the velocity of the fluid leaking out from the fracture face to the surrounding medium (i.e. the fluid velocity normal to the fracture face at the fracture wall). The point source $Q_{i n j}$ on the right-hand side of the conservation equation models the injection from a wellbore, the size of which is presumed negligible in comparison to the fracture size. Extension to account for a finite size wellbore is straightforward.

Fracturing fluids (which are typically in liquid phase) have a low compressibility. Under isothermal conditions, the fluid equation of state can thus be linearized $(\rho \approx$ $\rho_{o}\left(1+c_{f}\left(p-p_{o}\right)\right)$, where $\rho_{o}$ is the fluid density at the reference pressure $p_{o}$ and $c_{f}$ the fluid compressibility) and the mass conservation re-written as

$$
\partial_{t} w+c_{f} w \partial_{t} p+\partial_{s_{i}}\left(w V_{i}\right)+2 v_{L}=Q_{i n j}(t) \delta\left(x-x_{i n j}\right)
$$

recognizing that $c_{f}\left(p_{p} o\right) \ll 1$ for typical value of the fluid compressibility (in $O\left(10^{-10}\right) P a^{-1}$ ) and fluid pressure variations (in $O\left(10^{6}\right) P a$ ). Moreover, as soon as the fracture is open, its elastic compliance is much larger than the elastic compliance of the fluid, that is, $1 / w \partial_{t} w \gg c_{f} \partial_{t} p$. This can be easily grasped as $w \propto p / E \times L$, where $L$ is a fracture characteristic length. The mass conservation for a fracturing liquid therefore further reduces to volume conservation:

$$
\partial_{t} w+\partial_{s_{i}}\left(w V_{i}\right)+2 v_{L}=Q_{i n j}(t) \delta\left(x-x_{i n j}\right) .
$$


Integration over the entire fracture surface from the beginning of pumping to the current time $t$ results in the global volume conservation

$$
\mathcal{V}_{\text {fracture }}+\mathcal{V}_{\text {leak-off }}=\mathcal{V}_{\text {inj }}
$$

where $\mathcal{V}_{\text {fracture }}, \mathcal{V}_{\text {leak-off }}$ and $\mathcal{V}_{\text {inj }}$ are the fracture, total leaked-off and injected volume respectively. This global volume conservation is valid in the case of a vanishingly small fluid lag (see section 2.1.5 for discussion on the vanishing size of the lag for sufficiently deep fracture).

For an incompressible fluid, one obtains the following width-averaged balance of momentum for the fluid (in the fracture plane):

$$
\rho\left(\partial_{t} V_{i}+V_{j} \partial_{s_{j}} V_{i}\right)=-\partial_{s_{i}} p-\frac{2}{w} \tau_{w}
$$

where $\tau_{w}$ is the time-averaged fluid shear stress. The inertial terms on the left hand side are negligible in all cases for a fracturing liquid except at extremely early time (see discussions in Garagash (2006a); Zia and Lecampion (2017)). The fluid wall shear stress is typically expressed as

$$
\tau_{w}=f(R e, k / w) \times \frac{\rho V_{i}\|V\|}{2}
$$

where $f$ is the Fanning friction factor. It is a function of both the local Reynolds number of the flow $R e=\rho D_{e q}\|V\| / \mu$ (where $D_{e q}$ is an equivalent hydraulic radius for plane flow) and the fracture relative roughness $k / w$ (where $k$ is a characteristic scale of the fracture roughness - typically related to the rock grain size). As first discussed in Jones (1976), the definition of the equivalent hydraulic radius can be directly obtained such that the laminar parallel plate flow relation (Poiseuille law) for a Newtonian fluid is recovered when using the laminar expression of the Fanning factor for pipe flow $f=16 / R e_{D_{e q}}$, i.e. $D_{e q}=4 / 3 w$. A number of models exist for the evolution of the Fanning factor from laminar to turbulent flow in pipes, most are based on a combination of theory and phenomenological relations to capture experimental observations (Nikuradse, 1950). One should always treat those turbulent models with great care and recognize the spatio-temporal statistics associated with the transition to turbulence (Manneville, 2016). It is also extremely important to bear in mind that in industrial practice, friction reducers (Virk, 1975) are always added to low viscosity fluid in order to reduce drag under turbulent conditions. These polymer additives drastically modify the transition to turbulence and significantly lower the value of the friction factor in the turbulent regime (see discussions in Zia and Lecampion (2017)). 
When laminar flow conditions are encountered, which can be shown to apply in a majority of cases in industrial practice Zia and Lecampion (2017), the previous width-averaged balance of fluid momentum reduces to the well-known Poiseuille law between parallel plates, relating the fluid flux $q_{i}=w V_{i}$ to the local pressure gradient as

$$
q_{i}=w V_{i}=-\frac{w^{3}}{12 \mu} \partial_{s_{i}} p .
$$

where $\mu$ is the fracturing fluid viscosity. This so-called cubic law is valid for a fracture with an opening sufficiently large compared to the aperture roughness (Witherspoon, 1980) which is typically the case for hydraulic fracture.

\subsubsection{Carter's leak-off}

The leak-off rate $v_{L}$ from a hydraulic fracture located along $y=0$ and spanning for example $-\ell(t)<x<\ell(t)$ is typically modelled as

$$
v_{L}=-\left.\frac{k}{\mu} \frac{\partial p_{\Delta}}{\partial y}\right|_{y=0}
$$

where $p_{\Delta}=p_{r}-p_{o}$, the difference between the fluid pore pressure in the rock $\left(p_{r}\right)$ and the far-field (virgin) pore pressure $\left(p_{o}\right)$, evolves according to the diffusion equation

$$
\partial_{t} p_{\Delta}(x, y, t)=\frac{k}{\phi c_{r} \mu}\left(\partial_{x^{2}} p_{\Delta}(x, y, t)+\partial_{y^{2}} p_{\Delta}(x, y, t)\right)
$$

subject to

$$
\begin{aligned}
& p_{\Delta}(x, 0, t)=p(x, t)-p_{o}, \quad-\ell(t)<x<\ell(t) \\
& \lim _{y \rightarrow \infty} p_{\Delta}(x, y, t)=0 \\
& p_{\Delta}(x, y, 0)=0
\end{aligned}
$$

Here $k$ is the permeability of the rock, $\mu$ is the viscosity of the fluid within the hydraulic fracture, $c_{r}$ is the reservoir compressibility (combination of reservoir fluid and pore compressibility), $\phi$ is the porosity of the rock, and $p$ is the fluid pressure in the fracture and $p_{o}$ the far-field reservoir pore-pressure.

This 2D or 3D diffusion equation with time dependent boundary conditions that involve the fluid pressure $p$ - which is a part of the solution to the coupled hydraulic fracturing problem as well - poses a significant modeling challenge. The most common approach to overcoming this challenge is to follow the approach of 
Carter (Howard and Fast, 1957), who introduces two major simplifying assumptions. The first assumption is that the hydraulic fracture propagates much faster than the characteristic diffusion velocity. This assumption has been shown to be violated in high permeability formations (Kovalyshen, 2010). However, when valid, this assumption allows reduction to a $1 \mathrm{D}$ diffusion equation.

The second assumption is that the fluid net pressure $\left(p_{n e t}=p-\sigma_{o}\right)$ is very small compared to the far-field (virgin) effective stress, $\sigma_{o}-p_{o}$. By neglecting the net pressure under this assumption $\left(p-p_{o}=p-\sigma_{o}+\sigma_{o}-p_{o} \approx \sigma_{o}-p_{o}\right.$, the boundary conditions become uncoupled from the transient net pressure $p-\sigma_{o}$. Hence the diffusion problem reduces to

$$
\begin{array}{r}
\partial_{t} p_{\Delta}(x, y, t)=\frac{k}{\phi c_{r} \mu} \partial_{y^{2}} p_{\Delta}(x, y, t) \\
p_{\Delta}(x, 0, t)=\sigma_{o}-p_{o}, \quad-\ell(t)<x<\ell(t) \\
\lim _{y \rightarrow \infty} p_{\Delta}(x, y, t)=0 \\
p_{\Delta}(x, y, 0)=0
\end{array}
$$

which is readily solved to give

$$
p_{\Delta}=\left(\sigma_{o}-p_{o}\right) \operatorname{erfc}\left(\frac{\phi c_{r} \mu}{k} \frac{y}{2 \sqrt{t}}\right)
$$

Substitution into Eq. (13) leads to

$$
v_{L}=\frac{C_{c}}{\sqrt{t}}, \quad C_{c}=\sqrt{\frac{k c_{r} \phi}{\pi \mu}} \Delta p_{c}, \quad \Delta p_{c}=\text { const. } \approx \sigma_{o}-p_{o}
$$

Carter's leakoff model is then completed by two additional steps:

1. Introducing the dependence on the moving domain upon which the pressure boundary condition is applied, that is, $-\ell(t)<x<\ell(t)$, by replacing time $t$ in Eq. (22) with the fluid contact time $t-t_{o}(x)$, where $t_{o}(x)$ is the time it takes for the fluid to reach point $x$.

2. Generalizing to allow the fluid loss rate to potentially also be controlled by the need to displace reservoir fluid that has a different viscosity to the fracturing fluid and/or by the building of a low permeability filter cake comprised of particulate or polymer material carried by the fracturing fluid and left behind as the fluid infiltrates into the rock. 
This latter generalization leads to possible contributions of two additional processes with similar form to Eq. (22), but with different physical constants determining the coefficient $C_{c}$. However, because these generalizations also lead to $v_{L} \propto(t-$ $\left.t_{o}(x)\right)^{-1 / 2}$, the effects can be lumped into a single, composite fluid loss constant $C_{L}$, which is typically determined from calibration experiments such as diagnostic fracture injection tests (Nolte, 1979; Castillo, 1987; Barree and Mukherjee, 1996) and/or matching model predictions to fracture geometries inferred from microseismic monitoring (Weng et al., 2011b). The resulting form of Carter's leakoff equation is thus given by

$$
v_{L}(x)=\frac{C_{L}}{\sqrt{t-t_{o}(x)}}
$$

\subsubsection{Boundary conditions}

The propagation of hydraulic fractures is typically driven by a given fluid injection rate from a wellbore which can be modeled as a point source in the fluid continuity equation (9) (or eventually a line source if there are a line of perforations in a vertical well for example).

A lag region filled either with vapour (at cavitation pressure) or pore fluid (at reservoir pore pressure) respectively for an impermeable or a porous material may be present at the fracture tip. In this case, where the fluid front $\mathcal{C}_{f}$ lags behind the fracture front $\mathcal{C}$, the boundary conditions at the fracture front $\mathcal{C}_{f}$ are (for an impermeable medium)

$$
p=p_{\text {cav }} \approx 0 \text { and the Stefan condition } V_{i}=q_{i} / w
$$

Meanwhile, the boundary condition at the fracture front $\mathcal{C}$ is $w=0$ in complement to the propagation condition $K_{I}=K_{I c}$. It has been shown (Garagash and Detournay, 2000) that the fluid and fracture fronts actually coalesce when

$$
\frac{\sigma_{o} K_{I c}^{2}}{\mu V_{C} E^{\prime 2}} \gg 1
$$

for an impermeable medium where $V_{C}$ denotes the fracture tip velocity, $E^{\prime}=E /(1-$ $\left.\nu^{2}\right)$ is the rock plane-strain elastic modulus and $\sigma_{o}$ is the minimum in-situ stress acting perpendicular to the fracture (see Detournay and Garagash (2003) for the case of a porous medium). This condition for negligible lag is almost always fulfilled in practical applications at sufficient depth (i.e. above one hundred meters). For planar radial and plane-strain hydraulic fracture, the coalescence of the fluid and 
fracture front under constant injection rate happens over a timescale $t_{*}=12 E^{\prime 2} \mu / \sigma_{o}^{3}$ (Bunger and Detournay, 2007; Lecampion and Detournay, 2007), which is of the order of a few seconds or less for typical values of confining stress at depth, rock and fluid properties. It can be shown that when the fluid and fracture front coalesces, the Stefan condition degenerates to $q_{i}=0$ Detournay and Peirce (2014) and three conditions must be satisfied at all points along the fracture front $\mathcal{C}$ when the fracture propagates

$$
w=0 \quad q_{i}=0 \quad K_{I}=K_{I c}
$$

Under mixed mode loading, an additional criteria for the curving of the fracture is required, e.g. the maximum tensile stress criteria (Erdogan and Sih, 1963).

\subsubsection{Multiscale tip asymptotics}

The coupling of linear elastic fracture mechanics, elasticity, and lubrication flow leads to a complex solution near the tip of a propagating hydraulic fracture, which in turns leads to numerical difficulties as first recognized by Spence and Sharp (1985). It is only over the past 20 years that this tip structure of the solution has been recognized to play a critical role in the overall solution (see e.g. Garagash (2009)). Garagash and Detournay (2000) argue that the tip behavior is governed by local plane strain conditions, provided the crack front curvature is not too large. For a hydraulic fracture growing with velocity $V$, the continuity Eq. (9) can then be expressed for the tip region in terms of a moving coordinate attached to the crack tip $\hat{x}=V t-x)$ as

$$
\frac{\partial w}{\partial t}=\frac{\mathrm{D} w}{\mathrm{D} t}+V \frac{\partial w}{\partial \hat{x}}
$$

The key point is that the quantity $\partial w / \partial \hat{x}$ becomes large in the vicinity of the tip, and as a result the advective term $V \partial w / \partial \hat{x}$ dominates the time derivative $\mathrm{D} w / \mathrm{D} t$. Hence, the solution near the tip of a propagating finite hydraulic fracture is captured by a stationary solution for a semi-finite crack moving at a constant velocity $V-$ a solution akin to a traveling wave solution. For more details, we refer to the contributions of Desroches et al. (1994) for the zero toughness / zero leak-off case (viscosity asymptote), Lenoach (1995) for the leak-off dominated case and Garagash et al. (2011) for the complete solution with toughness, leak-off and viscosity in the limiting case of a vanishing lag.

It is instructive to re-emphasize here the structure of this tip solution in the case of a vanishing lag (where 24 is valid, i.e. for a deep fracture). Figure 2 displays the scaled fracture width with respect to the scaled distance to the fracture tip for 
different values of leak-off. Near the fracture tip $\left.\left(\hat{x} / \ell_{k m} \lesssim 10^{-3}\right)\right)$, the classical linear elastic fracture mechanics (LEFM) asymptotic $\left(w \propto x^{1 / 2}\right)$ is valid while away from the tip an "outer" viscosity asymptote appears $\left(\hat{x} / \ell_{k m} \gtrsim 10^{-1}\right)$. The effect of leak-off appears as an intermediate asymptotic between the linear elastic fracture mechanics and viscosity asymptote as a function of a dimensionless number

$$
\chi=\left(2 C^{\prime} E^{\prime}\right) /\left(K^{\prime} V^{1 / 2}\right)
$$

, i.e. a quantity determined by the leak-off coefficient, elastic plane-strain modulus, fracture velocity and toughness. One can easily anticipate that in a numerical model using a classical algorithm based on linear elastic fracture mechanics, the mesh size must be sufficient to capture the region where the LEFM asymptote is valid. In that respect, the actual value of the lengthscale $\ell_{m k}=K^{\prime 6} /\left(E^{\prime 4} \mu^{\prime 2} V^{2}\right)$, which scales the physical distance from the tip on Fig. 2, is particularly important. The condition on the mesh size to capture the LEFM region will become more stringent as $\ell_{m k}$ decreases.

As an example, let's assume the following realistic rock properties: $E^{\prime}=25 \mathrm{GPa}$, $K^{\prime}=\sqrt{32 / \pi} K_{I c}=\sqrt{32 / \pi} \times 1.5 \mathrm{MPa} \cdot \mathrm{m}^{1 / 2}, C^{\prime}=2 C_{L}=2 \times 10^{-5} \mathrm{~m} \cdot \mathrm{s}^{1 / 2}$ and fracture velocity $V=0.1 \mathrm{~m} / \mathrm{s}$. For these values, we obtain a small value for the leak-off dimensionless parameter $\chi=0.47$. For such a small value of $\chi$, the asymptotics are close to the zero leak-off case. In the case where the fracturing fluid is water $\left(\mu^{\prime}=\right.$ $12 \times 10^{-3} \mathrm{~Pa}$.s), we obtain 5350 meters as an estimate of the transition lengthscale $\ell_{m k}$. According to Fig. 2, the LEFM region (where the square root asymptote is valid) is thus of the order $10^{-3} \ell_{m k} \approx 5.3$ meters. On the other hand, if the fracturing fluid is akin to a linear gel for which $\mu^{\prime} \approx 12 \times 0.5 \mathrm{~Pa}$.s, we obtain $\ell_{m k}=0.021$ meters and thus a size of the LEFM region of the order of 21 microns. Such a simple example illustrates the drastic reduction in the size of the LEFM region for the case of viscous fluid (or equivalently faster fracture, stiffer material or/and lower toughness material). The requirement on the mesh size to capture the LEFM region (and therefore obtain an accurate numerical solution) can sometimes become prohibitive. This effect is intrinsically related to the multiscale nature of the hydraulic fracture solution near the fracture tip. It must be well understood in order to devise robust numerical schemes being able to accurately simulate the process over the whole possible range of values of the problem parameters (fluid viscosity, toughness etc.).

\subsection{Model Extensions}

A number of extensions to the base model previously described have been investigated in the literature. A few of the most often considered extension are briefly 


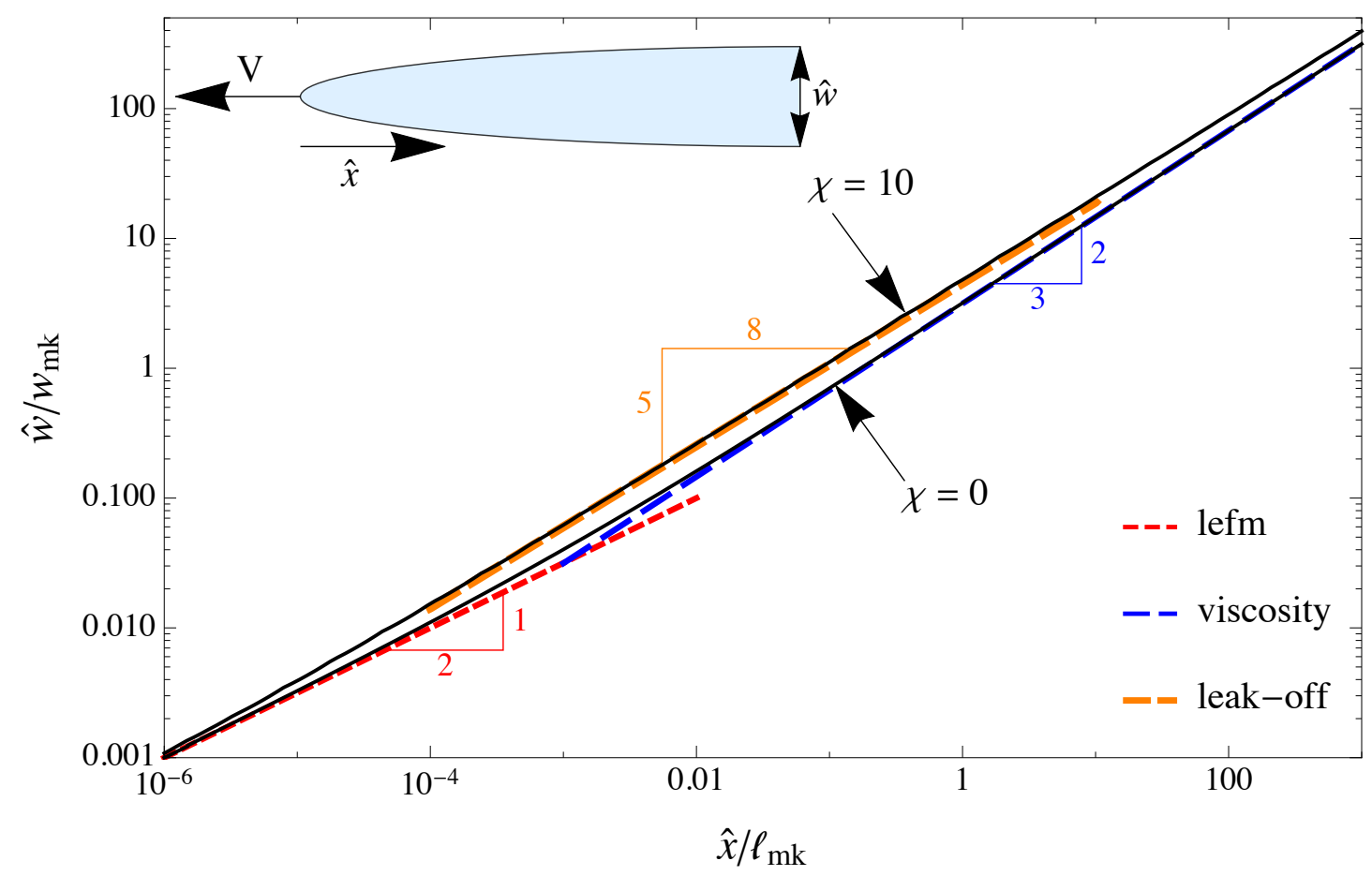

Figure 2: Dimensionless width $\hat{w} / w_{m k}$ with dimensionless distance from the tip $\hat{x} / \ell_{m k}$ for a semi-infinite plane-strain hydraulic fracture propagating at a constant velocity $V$ (Newtonian fluid). The characteristic width and length scales are given respectively by $w_{m k}=K^{\prime 4} /\left(E^{\prime 3} \mu^{\prime} V\right)$ and $\ell_{m k}=K^{\prime 6} /\left(E^{\prime 4} \mu^{\prime 2} V^{2}\right)$, where $K^{\prime}=\sqrt{32 / \pi} K_{I c}, \mu^{\prime}=12 \mu$ are related to fracture toughness and fluid viscosity, and $E^{\prime}$ is the rock plane-strain modulus. In the absence of leak-off $(\chi=$ $\left(2 C^{\prime} E^{\prime}\right) /\left(K^{\prime} V^{1 / 2}\right)=0$, where $\left.C^{\prime}=2 C_{L}\right)$, the near-tip width evolves from the linear elastic fracture mechanics (LEFM) square-root dependence close to the tip (for $\left.\hat{x} / \ell_{k m} \lesssim 10^{-3}\right)$ to the viscosity asymptote (2/3 dependence) away from the tip (for $\left.\hat{x} / \ell_{k m} \gtrsim 10^{-1}\right)$. For $\chi=10$, the leak-off asymptote (5/8 dependence of width with distance from the tip) appears as an intermediate asymptote, while the square-root LEFM asymptote shrinks to a very small region close to the tip $\left(\hat{x} / \ell_{k m}<\lesssim 10^{-6}\right)$, and the viscosity asymptote is only valid far-away from the tip $\left(\hat{x} / \ell_{k m} \gtrsim 10^{4}\right)$. See Garagash et al. (2011) for a complete discussion of the solution and its inner and outer asymptotes, we refer to Bunger and Detournay (2008) for experimental validation in the zero leak-off case. 
reviewed here.

\subsubsection{Poroelasticity}

Instead of utilizing Carter's leak-off approximation, one can instead simulate the reservoir as a porous medium and couple the equations of poroelasticity with the flow in the deformable hydraulic fracture (Boone and Ingraffea, 1990; Carrier and Granet, 2012). Such an approach is, of course, attractive from a theoretical point of view as it does not rely on the assumptions behind Carter's leak-off. In the limit of negligible poroelastic coupling and small rock diffusivity (compared to the injection duration), the results obtained are similar to the Carter's leak-off model (Carrier and Granet, 2012; Kovalyshen and Detournay, 2013). Poroelastic effects are obviously important when the injection duration is similar to the rock diffusion time-scales. Due to the pore pressure increase in the surrounding of the fracture, a poroelastic back-stress develops resulting in an increase of the fracturing pressure and shorter fracture extension.

In practice, as already mentioned in sub-section 2.1.4, a "skin" always builds up on the fracture walls. Such a skin build-up can be explicitly modeled although, similar to Carter's leak-off model, the coefficient(s) appearing in such a description are rather ad-hoc, fluid chemistry dependent, and must be determined experimentally. For industrial applications related to well stimulation, the need to use a complete poroelastic model is therefore questionable. Prior to complexifying the modeling, depending on the reservoir and injection condition, the scaling laws derived in Kovalyshen and Detournay (2013); Kovalyshen (2010) should be used in order to gauge of the relevance of such a modeling. However, poroelastic effects must be addressed to model fracture closure upon the end of injection. Multiphase flow effects must also obviously be accounted for to properly model flow-back and production from a hydraulic fracture. It should also be noted that classical poroelasticity based on the assumption of a rock that is saturated by a single phase liquid may also be inappropriate for use in many gas reservoirs for which the partial gas saturation can be in the order of tens of percent.

\subsubsection{Fluid non-linearities}

A large number of fracturing fluids used in practice have a non-Newtonian behavior; see Barbati et al. (2016) for a review of fracturing fluids. Although liquefied gas is sometimes (but seldom) used, most of the fracturing fluids are polymer aqueous solutions. These polymeric fluids typically exhibit shear-thinning properties, i.e. their 
viscosity decreases with shear-rate. Such types of fluid rheology can be modelled by a power-law viscosity relationship for which an analytical solution equivalent to Poiseuille's law (12) can be easily obtained. A more refined rheological description of any fracturing fluid should account for a limiting value of its viscosity at both low and large shear rates using for example the Cross (1965) or Carreau (1972) rheological models. Some of the fluids used in the industry also exhibits viscoelastic properties, which are popular because these greatly enhance the ability of the fluid to carry solids (proppant) particles. It is worth mentioning that these complex fluids are typically sufficiently viscous such that the flow is always laminar within the fracture.

The addition of proppant to the carrier fluid modifies the slurry rheology. A proper two-phase (carrier fluid and solid particles) constitutive model is needed to properly describe the flow of such suspensions in fracture (Lecampion and Garagash, 2014). After averaging across the fracture width at a given location, the effect of solid concentration on the flow of such suspension can be accommodated by a tangent viscosity function of the solid volume fraction while the conservation of mass of the solid particles adds a coupled transport-like equation on top of the slurry continuity equation (see Chekhonin and Levonyan (2012); Dontsov and Peirce (2015b) for examples of numerical implementation of such type of models). We refer to Osiptsov (2017); Hormozi and Frigaard (2017) for a recent discussion of a wide range of issues related to the modeling of proppant transport in hydraulic fracturing.

\subsubsection{Solid non-linearities}

Extensions to account for the bulk plasticity of the rock have been addressed by several authors (Papanastasiou and Thiercelin, 1993; Papanastasiou, 1997; Sarris and Papanastasiou, 2013). Bulk plasticity results in an apparent toughening of the rock. The necessity of such an added modeling complexity is obviously dependent on the type of reservoir rock. Although, it appears critical for so-called "frack and pack" applications in unconsolidated reservoirs, it will be of second order for brittle reservoir rock (Germanovich et al., 2012).

The solid non-linearites associated with fracture propagation in a quasi-brittle material like rocks are often captured by cohesive zone models. A large number of cohesive zone models are available (see Park and Paulino (2011); Needleman (2014) for review). These models condense all the solid non-linearities along the fracture surface via a traction-separation constitutive law. They model the fact that the fracture energy $G_{c}$ is spent over a "process zone" lengthscale. Cohesive zone models combining opening and shear fracture mode correctly are capable of 
reproducing mode $\mathrm{I}+\mathrm{II}$ fracture growth but are unable to reproduce the splitting of the fracture front occurring under combined mixed I+III opening / anti-plane shear modes. Cohesive zone models are numerically attractive due to their simplicity of implementation but the mesh resolution must be sufficient in order to capture the fracture process zone.

\subsubsection{Non-linear injection conditions}

The condition of an imposed, known, flow rate $Q_{o}(t)$ at the inlet of the fracture does not always hold. In particular, in the early stage of fracture growth, the flow rate entering a fracture may be strongly influenced by the injection system compliance (see Lhomme et al. (2005); Lakirouhani et al. (2016); Lecampion et al. (2017) for detailed discussions of this effect). This is also the case where multiple hydraulic fractures located at different positions along a well propagate simultaneously due to a constant injection rate in the wellbore. In that case, the exact splitting of the fluid injected at the well head into the different fractures is a priori unknown and may evolve with time. It depends notably on the elastic interactions between the different fractures as well as the exact characteristics of the flow constrictions between the fractures inlet and the wellbore - so called perforation friction (see Bunger and Peirce (2014); Lecampion and Desroches (2015b); Lecampion et al. (2015) for some examples). The propagation of simultaneous multiple fractures from a wellbore therefore adds another non-linearity which must be properly dealt with by coupling the dynamics of the fluid flow in the wellbore with the growth of these multiple hydraulic fractures (see Lecampion and Desroches (2015a) for the description of a fully implicit scheme for such problem).

\section{The challenges of a numerical solution and the different schemes}

A large variety of numerical methods have been proposed to model hydraulic fracture growth. We will not attempt to exhaustively classify all of them. However, a distinction between numerical schemes can be broadly made from the way the fracture front is modelled and how the fracture propagation is handled. A first family of schemes explicitly tracks the fracture front at each given time step. This is notably the case of most of the schemes based on linear elastic fracture mechanics. It does not necessarily imply that the fracture front always exactly coincides with the mesh boundary although this is often the case. 
A second family of schemes "captures" the location of the fracture front. In other words, the position of the fracture front is resolved up to the spatial discretization size of the scheme. This is notably the case of algorithms using cohesive zone models within finite element analysis, where the fracture follows cohesive zone elements. It is also the case of meso-scale models like the ones based on the distinct element method (or quasi-random lattice models). These types of schemes are usually simpler to develop with respect to fracture propagation but typically require a finer mesh discretization to capture the fracture process zone accurately. The accuracy of the time evolution of the fracture footprint is intrinsically related to the spatial discretization for those schemes. The numerical algorithms based on a phase field approach to fracture also falls into such an interface capturing category as the fracture is typically "smeared" over a number of elements in those methods.

It is worth noting that for some simple fracture geometries (planar fracture in plane-strain or axisymmetry), a moving mesh approach is very efficient (Desroches and Thiercelin, 1993; Lecampion and Detournay, 2007). In such approach, the fracture domain is re-scaled to the interval $[-1,1]$ and the fracture length solved as an additional scalar variable.

\subsection{Solution of the elasto-hydrodynamics system}

Different numerical techniques can be used to solve the coupled system of equations arising from the coupling between elastic deformation and lubrication flow in the fracture. The elasticity equation can be discretized via finite element or boundary element methods, while finite elements, finite volumes or finite differences are typically used to discretize the lubrication flow in the fracture. As discussed in Adachi et al. (2007), in all cases the resulting CFL (Courant Friedrichs Lewy) condition on the time step $\Delta t$ for the stability of the coupled elasto-hydrodynamics system scales as $\Delta t<h^{3} / E^{\prime} D$ (where $h$ is the mesh size and $D \approx \bar{w}^{3} / \mu$ is a diffusivity which is a function of a nominal fracture width $\bar{w}$ ). Implicit time-stepping schemes (i.e. backward Euler) are thus clearly favored for the solution of this coupled system of equations.

For a given time-step $\Delta t$, using an implicit time integration scheme, the resulting system of equations is non-linear and extremely stiff. This is a direct consequence of the relation between the fracture conductivity and the cube of the fracture opening for a Newtonian fluid. Such a non-linear system can be solved either via fixed point iterations or quasi-Newton methods (see e.g. Quarteroni et al. (2000) for a textbook description). For more robustness, a quasi-Newton method can be started after few fixed point iterations have been performed. In doing so, one benefits from a "good" 
starting guess for the quasi-Newton method. Adequate pre-conditioners for the solution of the quasi-Newton Jacobian residuals system have been investigated in Peirce and Siebrits (2005); Peirce (2005), with ILU based pre-conditioners showing the best compromise. Note that pinch-points (i.e. points where the fracture faces contact themselves locally) may develop in the presence of in-situ stress jumps and elastic modulus variations. An appropriately-chosen solver tackling such width constraints must thus be used (Peirce and Siebrits, 2005; Peirce, 2005).

It is important to bear in mind that the solution of the elasto-hydrodynamics system of equations over a given time-step is typically performed for a given fracture footprint. This non-linear system must thus be solved a number of times for a number of "trial" locations of the new fracture footprint, hence its computational performance is critical.

\subsection{Fracture front tracking}

The way the new position of the fracture front is solved for over a time-step is critical with respect to the accuracy of the solution of the problem. Different strategies are possible, and the choices often define the type of algorithms. As aforementioned, "capturing" schemes are typically simpler to develop but resolve the fracture front up to the spatial discretization.

In the case where the fracture front is "tracked", its new position can be obtained either explicitly or implicitly. Explicit methods update the new fracture front at time $t_{n}+\Delta t$ from the velocity of the fracture front at time $t_{n}$ (and its propagation direction if under mixed mode loading). Such explicit fracture front propagation methods are often used in "dry" fracture mechanics problems, where ad-hoc laws are sometimes adopted to estimate the local fracture velocity from the local value of stress intensity factors, using for example Paris' fatigue law which can be seen as a regularization of brittle fracture (Lazarus, 2003). In the case of hydraulic fracturing, the fracture velocity results from the strong fluid-solid coupling at play in the near tip region and the use of such empirical growth laws are not warranted.

The elasto-hydrodynamics system of equations has a severe CFL condition (in $O\left(h^{3}\right)$ where $h$ is the mesh size). A fully explicit scheme for both fracture front position and the elasto-hydrodynamics system is therefore a poor choice if the fracture front is "tracked" because the small time step requirements implies small increment of the fracture front, smaller mesh size associated with the small fracture increment results in a smaller critical time step, and so forth thus leading to a vicious cycle of refinement of the length and time discretizations. The elasto-hydrodynamics system is thus typically solved implicitly avoiding the restriction on time-step as- 
sociated with the CFL condition, while the fracture front is still evolved explicitly. There are no real guidelines for the choice of the time-step ensuring stability for such hybrid schemes. Such a implicit (for fluid-solid coupling) / explicit (for fracture front increment) scheme will perform poorly in the presence of heterogeneities and/or in-situ stress jumps if the time-step is too large, because the fracture front position is estimated from quantities known at the end of the previous time-step.

The alternative is a fully implicit scheme, both for the position of the fracture front and the solution of the elasto-hydrodynamics system (i.e. for a given position of the fracture front). An implicit scheme to track the fracture front thus necessitates iteration on the new fracture front, i.e. a number of trial positions of the fracture front will be generated and the elasto-hydrodynamic coupling needs to be solved on this trial domain. Such types of implicit scheme will typically consist of two nested loops over a time-step: 1) an outer loop for the new location of the fracture front, and 2) an inner loop solving for the elasto-hydrodynamics system (for every trial position of the new fracture front). Such types of schemes are typically more accurate and robust meaning that larger time-steps can be taken. However, their developments are more intricate and a fast elasto-hydrodynamics solver is a must for computational efficiency.

The treatment of fracture curving under mixed mode loading can be tackled with method developed for dry fracture propagation. Typically, it is easier to use an explicit treatment of the fracture front advance under such type of mixed mode loading. However, one can also specify explicitly the direction of propagation from quantities computed in the previous time-step and implicitly calculate the increment of the fracture advance in the set direction.

Another very important point worth re-emphasizing regards the multiscale structure of the solution near the fracture tip and the mesh requirements associated with the use of conventional linear elastic fracture mechanics (LEFM). When using a scheme based on LEFM (i.e stress intensity factor based) to track the fracture front, the mesh needs to be sufficiently fine to capture the LEFM region which scales with $\ell_{m k}$ (See sub-section 2.1.6 for discussion).

\section{Advances in algorithms for continuum models}

In what follows, we review some of the new algorithms and numerical techniques developed for continuum models of hydraulic fracture growth before discussing mesoscale approaches in section 5 . 


\subsection{Schemes using the multi-scale hydraulic fracture tip asymp- totes for fracture front tracking}

In recent years, very efficient fully implicit schemes for the propagation of planar hydraulic fractures have emerged. These schemes use the asymptotic semi-infinite hydraulic fracture tip solution (see Fig. 2) coupled with a finite discretization of the rest of the fracture. In doing so, these schemes bypass the strong requirement on mesh size associated with the use of the linear elastic fracture mechanics propagation condition in the case of viscosity dominated propagation (as previously discussed). Extremely accurate and robust numerical solutions can thus be obtained even on coarse meshes. We very briefly outline here the basic idea of these schemes. A complete description of such implicit algorithms using either a boundary element or an extended finite element discretization of the elasticity equation can be found in a recent review (Peirce, 2016), see also Peirce and Detournay (2008); Peirce (2015); Dontsov and Peirce (2017) for details and examples.

In the implicit schemes, the finite fracture is divided in a "tip" region (few elements close to the fracture front) where the HF tip width solution holds and a "channel" region, i.e the rest of the fracture away from the tip. The coupling of the HF tip solution with a finite discretization of the fracture is performed via the elements at the boundary between the channel and tip regions - so-called "ribbon" elements. The opening of the ribbon elements is used to invert the complete $\mathrm{HF}$ tip asymptote and thus obtain a trial estimate of the closest distance between each ribbon element and the fracture front. It is important to point out that the fracture velocity which enters the HF tip solution is unknown and solved for during the process with such an implicit scheme. For planar 3D fractures, the fracture front is a level set function and a fast marching method is used to estimate the new fracture front position from the knowledge of the closest distance to the front at all the ribbon elements center. Once the new trial position of the fracture front has been obtained, the HF tip asymptotic solution is enforced in the tip elements (i.e. fixing the element volume according to the tip asymptotics) in order to complete the coupling with the rest of the fracture. For a given time-step, the process is repeated iteratively until convergence.

The results obtained with such a scheme compared extremely well with known semi-analytical solutions (Peirce and Detournay, 2008; Peirce, 2015). They have shown extremely good convergence properties when compared to other numerical codes (Lecampion et al., 2013).

A similar scheme can be used for $2 \mathrm{D}$ fracture in a much simpler way as the fracture front reduce to single points in that case (see Gordeliy and Peirce (2013b); 
Lecampion and Desroches (2015a)). The algorithm has also been extended to mixed mode I+II for plane-strain fractures. It is, however, unclear how it could be extended to non-planar 3D fractures exhibiting full mixed mode loading. A closely related scheme using the fluid velocity as a main variable and coupling the near-tip behaviour with a finite discretization of the rest of the fracture have been shown to perform well for plane-strain and height contained hydraulic fractures (Wrobel and Mishuris, 2013, 2015). It is also important to bear in mind that these algorithms assume that the fracture propagates (positive velocity). They can, however, be easily adapted to simulate fracture initiation (or cases where part of the fracture front does not propagate) by first checking for satisfaction of the propagation condition (see e.g. Lecampion and Desroches (2015a); Dontsov and Peirce (2017).

\subsection{Boundary element schemes}

For uniform or piece-wise uniform media (e.g. layered media) of infinite extent, as often encountered in applications, the boundary element method (BEM) has some clear advantages. The discretization of the elastic problem reduces to defining the unknowns only on the fracture surface, which in turn significantly lowers the size of the problem.

If a displacement discontinuity based BEM (DDM) is employed (Crouch and Starfield, 1983), the elasticity equations can be constructed readily by collocation through the fact that stresses along the fracture are equal to the superposition of the contributions of displacement discontinuities on all segments of the fracture. DDMbased methods have been widely used in simulating hydraulic fracturing in two and three dimensions for homogeneous as well as multi-layered medium ( see Vandamme and Curran (1989); Siebrits and Peirce (2002); Yamamoto et al. (2004); Lecampion and Detournay (2007); Gordeliy and Detournay (2010); Napier and Detournay (2013); Kumar and Ghassemi (2016) among others). Contact and friction along the fracture surface can also be readily detected through the changes of displacement

discontinuities with such type of methods. It can thus simulate the formation of complex fracture networks generated by hydraulic fracture propagation and the interaction between hydraulic fracture and natural fracture in two dimensions (e.g., Zhang et al. (2007); Weng et al. (2011a); Zhang and Jeffrey (2014)).

A number of other types of BEM-based formulation for crack problems have also been used in the hydraulic fracturing context. Among those, the Symmetric Galerkin BEM (SGBEM) is a conforming method to enforce variable continuity across element boundaries based on shape function like in the finite element method (see e.g.Bonnet et al. (1998) for more details). The SGBEM formulation with dis- 
placement discontinuities as the main variables for the discretization of the fracture have been used to solve for the elastic deformation in some 3D hydraulic fracture simulators (Rungamornrat et al., 2005; Xu and Wong, 2013). Recently, a domain modification BEM technique has been proposed in order to reduce the size of the elastic problem compared to a DDM or dual BEM discretization of the elastic problem (Cherny et al., 2016).

In all models using boundary elements for the solution of the elastic equation, the lubrication flow is discretized via either a finite volume (Peirce, 2005; Peirce and Detournay, 2009) or a finite element scheme (Advani et al., 1990). Finite volume methods are very efficient when coupled with DDM scheme for elasticity which do not enforce continuity of displacement discontinuity between elements. Galerkin finite element methods using continuous test functions have also been used, and are especially well suited when coupled with SGBEM. In all cases, the discretization results in a non-linear system of equations if an implicit time integration is used (see section 3.1 for discussion). The details of the fracture front propagation differ from scheme to scheme but usually involve the addition of new elements (Carter et al., 2000) and/or remeshing near the fracture front (Advani et al., 1990).

\subsection{Finite element schemes}

When the body shape is irregular, material properties are heterogeneous, and/or solid non-linearities are involved, the finite element method is often the method of choice to solve the mechanical problem. The simulation of fracture propagation in the framework of the finite element method can be performed with a large number of different approaches. For sake of discussion, we broadly classify them in four distinct categories as described in Fig. 3. The following provides an overview of each of these categories.

\subsubsection{Fracture growth with re-meshing}

One can use adaptive re-meshing during fracture growth such that the finite element mesh always exactly fits with the fracture surfaces (Simoni and Secchi, 2003; Secchi et al., 2007) (see Figure 3a). Such a strategy is, of course, computationally expensive especially in $3 \mathrm{D}$. The use of adaptive remeshing in the context of hydraulic fracturing simulation has recently received a renewed attention, see the recent work of Paluszny and Zimmerman (2011) for dry fracture, and Salimzadeh et al. (2016) for planar 3D hydraulic fractures. A local remeshing approach (i.e. limited only to the surrounding of the fracture front) has been developed in $3 \mathrm{D}$ and coupled to fluid flow in a 
staggered way with limited testing of the different hydraulic fracture propagation regimes (Profit et al., 2015, 2016a).

For multiphysics problems (e.g. poroelasticity etc.), the required interpolation of field variables between time-step associated with re-meshing is a particularly sensitive issue, especially in regards to mass conservation. Robust schemes for such interpolation are notoriously difficult to develop (Secchi et al., 2007).

\subsubsection{Growth along a pre-defined path}

When the fracture trajectory is known in advance such as for example for the case of a straight planar fracture (or T-shape/H-shape geometry where the fracture path is predetermined along few interfaces), the use of cohesive zone element to model fracture growth is relatively popular. Note that linear elastic fracture mechanics model also simplifies when the fracture path is predetermined, reducing the complexity of finding the fracture front within a priori known planes (Hunsweck et al., 2013; Golovin et al., 2015).

Cohesive zone models have been widely used for fracture nucleation and growth in elastic and poroelastic solids (Simoni and Secchi, 2003; Sarris and Papanastasiou, 2011, 2013; Yao et al., 2015; Carrier and Granet, 2012; Chen et al., 2009; Chen, 2012; Yao et al., 2015). The cohesive zone model originates from the theory proposed by Dugdale (1960) and Barenblatt (1962) to overcome the stress singularity ahead of the fracture tip. In the cohesive zone, a constitutive relation between the traction and the fracture surface separation and/or slip is controlled by a potential (Tvergaard and Hutchinson, 1992; Park and Paulino, 2011). For hydraulic fracturing problems, mode I crack propagation is typically assumed so that the contribution of shear stress on fracture energy is neglected. In order to accurately simulate both toughness and viscosity dominated hydraulic fracture propagation, a fine mesh is required to properly capture the process zone length scale (see Lecampion et al. (2013) for examples and discussions). The mesh needs to be even smaller than when using linear elastic fracture mechanics. And furthermore, the details of the cohesive constitutive law (tensile strength, maximum separation, softening shape etc.) are known to have an impact when the cohesive zone size is large (compared to the fracture size) (Needleman, 2014), see (Chen et al., 2009; Yao et al., 2015) for some discussions in the hydraulic fracturing context.

Extension to unknown fracture path Cohesive zone models can be generalized

to an unknown path by either inserting cohesive interface elements between all finite elements of the mesh (intrinsic approach), or inserting them on-the-fly when the 
interface between two elements reach its yield point (extrinsic approach). The latter technique - although more difficult to implement - is much less computationally expensive. In both cases, the fracture trajectory follows the mesh internal boundaries (see Fig. 3b). The fracture solution will thus always possess a degree of mesh dependency. Such a mesh dependency is of course minimized by using a random but uniform mesh with small element sizes in order to avoid propagation bias in some directions. However, whether a zig-zag fracture path can produce a reasonable fluid pressure distribution or not requires further investigation. Extrinsic type schemes have also been developed in the framework of linear elastic fracture mechanics $(\mathrm{Fu}$ et al., 2013; Settgast et al., 2016).

Combined Finite Element/ Distinct Element techniques (FEM/DEM) are closely related to the cohesive zone approaches just described; they usually involve an explicit time-stepping and an efficient node contact detection algorithm akin to discrete element method. The hydro-mechanical coupling essential to hydraulic fracturing has been investigated (Yan and Zheng, 2016), although no benchmarks with known solutions for hydraulic fracturing have been reported thus far.Another scheme (Profit et al., 2016a,b) combines FEM/DEM with a damage law in the bulk material and a local tip remeshing scheme such that the fracture propagation is not constrained to follow the initial mesh edges.

\subsubsection{Extended / Generalized finite element formulations}

The idea to separate the finite element mesh from the exact morphology of discontinuities such as fractures and dislocations dates back to the 1990s (Melenk and Babuška, 1996; Belytschko and Black, 1999; Moës et al., 1999). The method consists in enriching the classical finite element representation of field unknowns with an appropriately-chosen interpolation function and associated degrees of freedom. In the extended finite element method (XFEM), the enrichment is performed locally on the elements intersected by the discontinuity(ies) (see Fig.3c). For fractures, the displacement field is thus interpolated as:

$$
\begin{aligned}
\mathbf{u}= & \sum_{\forall I} N_{I}(\mathbf{x}) \mathbf{u}_{I}+\sum_{J \in S_{H}} N_{I}(\mathbf{x})\left[H(f(\mathbf{x}))-H\left(f\left(\mathbf{x}_{J}\right)\right)\right] \mathbf{q}_{J} \\
& +\sum_{i} \sum_{K \in S_{T}} N_{K}(\mathbf{x})\left[\Psi^{i}(f(\mathbf{x}))-\Psi^{i}\left(f\left(\mathbf{x}_{J}\right)\right)\right] \mathbf{q}_{K}^{i}
\end{aligned}
$$

where $H$ denotes the Heavyside function given by 1 for $f>1$ and 0 otherwise, $f$ is a level set function implicitly describing the fracture (for which $f(\mathbf{x})=0$ ) and 
a)

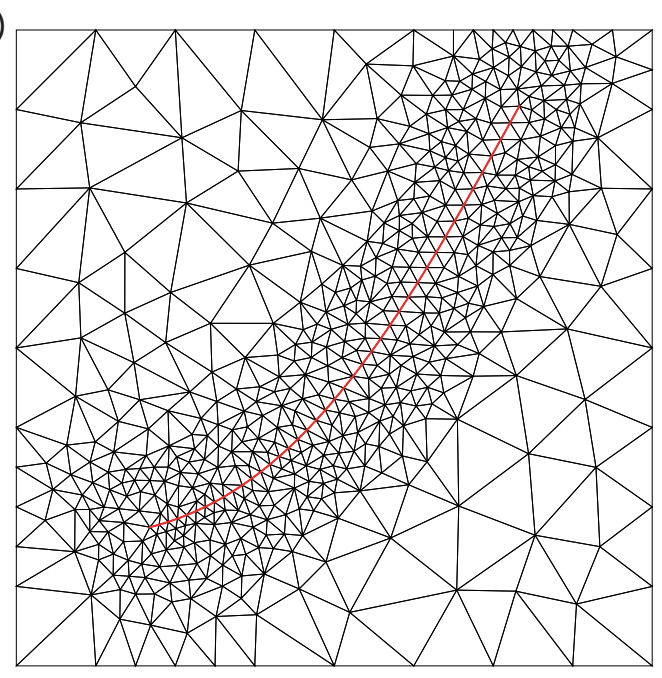

c)

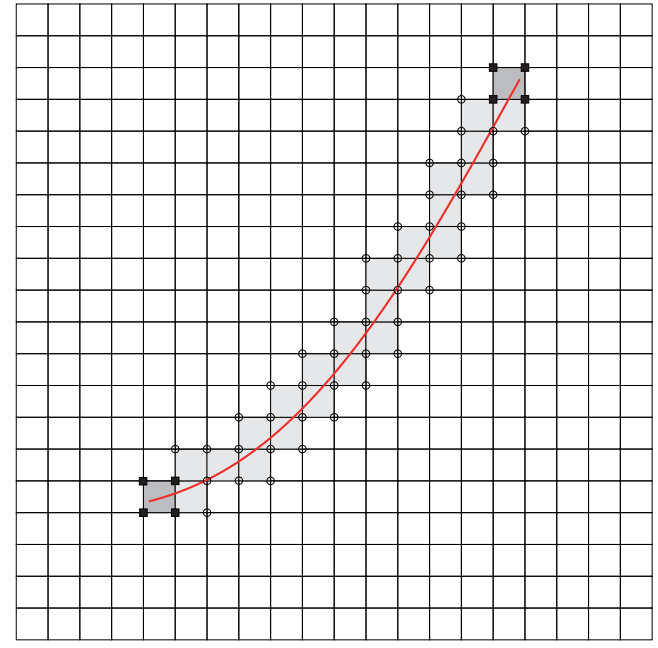

b)

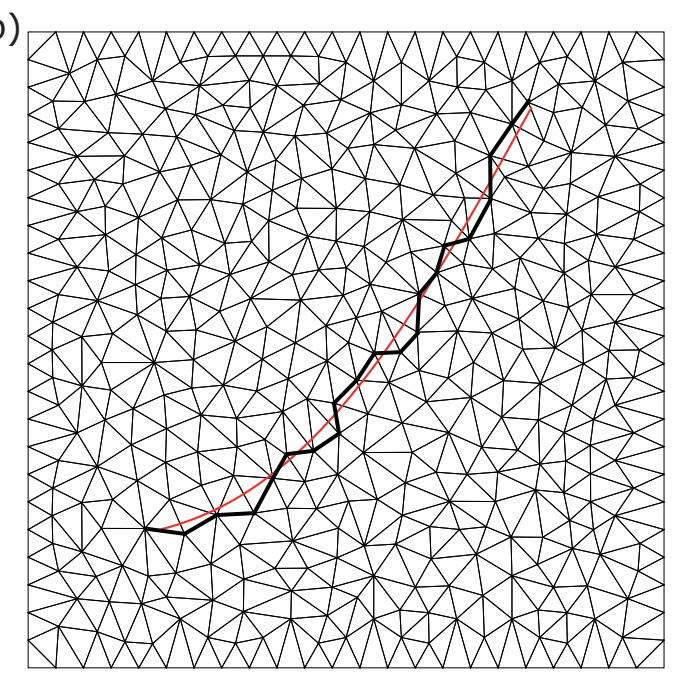

d)

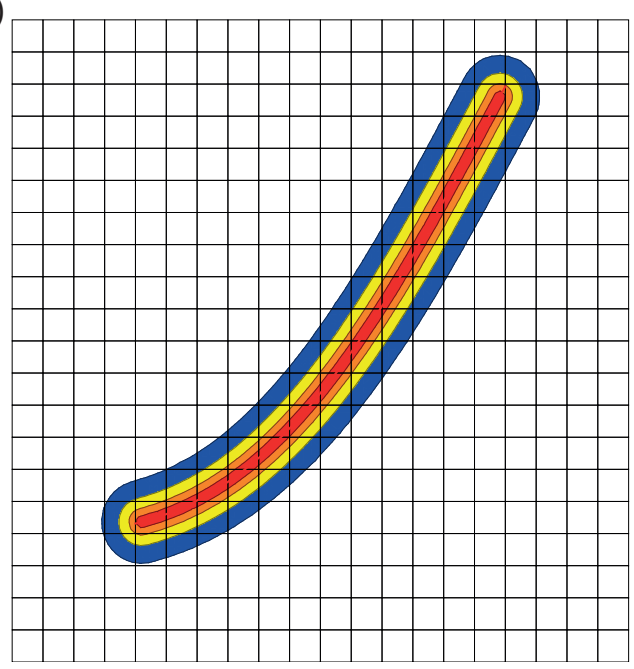

Figure 3: Four different categories of modeling fracture with finite elements ( The "real" fracture geometry in this figure is displayed in red): a) with adaptive remeshing (such that the mesh always fit the fracture faces), b) with cohesive zone elements (the approximated fracture geometry is black and follows element edges), c) with enriched degree of freedoms to model displacement discontinuities independently of the underlying mesh (XFEM), d) with a phase-field approach to fracture (zero damage in white for clarity). 
$\Psi^{i}$ are a set of enrichment functions approximating the near-tip behaviour of the solution. Additional degree of freedoms $\mathbf{q}_{J}, \mathbf{q}_{K}^{i}$ are associated with these additional interpolation functions on the set of elements $S_{H}$ and $S_{T}$ which are crossed by the fracture, with $S_{T}$ the set of elements near the fracture tip. Only those elements require special treatment when assembling the finite element stiffness matrix. The previous interpolation provides a local partition of unity essential in finite elements, although special care needs to be taken in the blending elements around the set of tip elements (see e.g.Belytschko et al. (2009) for more details). General discussions of the different aspects of the method in the context of solid mechanics can be found in Karihaloo and Xiao (2003); Belytschko et al. (2009); Fries and Belytschko (2010).

In order to address hydraulic fracturing problems, the lubrication flow inside the fracture needs to be discretized and coupled to elastic deformation in a consistent way. A number of different strategies can be adopted in order to solve for such a coupling (see Gordeliy and Peirce (2013a) for a complete discussion and the benefits of a mixed formulation). Another important point relates to the choice of the tip enrichment functions. As the tip asymptote(s) may differ from the classical LEFM case (see subsection 2.1.6 for details), different tip functions need to be used depending on the propagation regimes for better numerical performance (see Lecampion (2009); Gordeliy and Peirce (2015)).

As with other categories of finite element simulations, different schemes can be devised for tracking the fracture propagation. Implicit schemes using the knowledge of the hydraulic fracture tip behaviour have been successfully developed for 2D problems, properly reproducing semi-analytical solutions in the different propagation regimes without leak-off (Gordeliy and Peirce, 2013b).

Cohesive zone models can also be easily used with XFEM. In 2D, using an extension of XFEM accounting for poroelasticity, a cohesive zone approach within an XFEM formulation appears to properly reproduce the known solutions for a planestrain propagating hydraulic fracture (Faivre et al., 2016). See also Mohammadnejad and Khoei (2013a,b); Salimzadeh and Khalili (2015) for the fully or partially saturated case but with fewer benchmarks, and Mohammadnejad and Andrade (2016) for the case of fracture closure and re-opening. Extensions to account for frictional joints/fractures have also been proposed by Khoei et al. (2015). When using XFEM for fluid infiltrated medium, a proper enrichment needs to be implemented to reproduce the pore-pressure variation in the solid matrix associated with the fracture (discontinuity in the pressure gradient normal to the fracture). Different schemes have been proposed (Mohammadnejad and Khoei, 2013a; Faivre et al., 2016; Meschke and Leonhart, 2015). The lack of consistent benchmarking and convergence studies renders the discussion on the efficiency and robustness of these different schemes 
rather difficult. Also, the problem of resolving the junction(s) of different fractures (i.e. intersections etc.) is difficult in the classical XFEM framework, and properly handling the junction of several cracks via XFEM is known to be particularly difficult and requires the development of special techniques (see Karihaloo and Xiao (2003) for discussion).

Three dimensional modeling with XFEM is notoriously more challenging from an implementation point of view than the 2D case. In the limit of toughness dominated propagation (which implies a uniform distribution of fluid pressure), Gupta and Duarte (2014) have developed a formulation to model 3D mixed mode hydraulic fracture propagation, although conserving the continuity of the fracture front and therefore not fully reproducing the fracture front segmentation observed experimentally under mixed mode I+III. The formulation has been extended to account for the coupling with lubrication flow (Gupta and Duarte, 2015) and results for the case of a static fracture (no propagation) presented.

\subsubsection{Phase field methods}

The phase field approach to fracture consists in introducing a continuous scalar variable $s$ - the field order parameter or crack field - describing the smooth transition between the intact and the fully broken material state. In such a framework, the sharp displacement discontinuity associated with a fracture is approximated, typically smeared out over few elements (see Fig. 3d). The approach can be seen as a regularization of a variational formulation of brittle fracture (Francfort and Marigo, 1998). The fracture propagation (and initiation) problem can be seen as the solution of $E_{\ell}\left(u_{i}, s\right)=P\left(u_{i}\right)$, where $P$ is the work of external forces and $E_{\ell}$ is the following regularized energy functional (Bourdin et al., 2000), that is

$$
E_{\ell}\left(u_{i}, s\right)=\int_{\Omega}\left(s^{2}+k\right) \Psi\left(\epsilon_{i j}\left(u_{i}\right)\right) \mathrm{d} x+G_{c} \int_{\Omega}\left(\frac{1}{4 \ell}(1-s)^{2}+\ell\|\nabla s\|^{2}\right) \mathrm{d} s
$$

Here $\Psi$ is the classical elastic energy density function $\Psi\left(\epsilon_{i j}\left(u_{i}\right)\right)=1 / 2 \epsilon_{i j}\left(u_{i}\right) C_{i j k l} \epsilon_{k l}\left(u_{i}\right)$ and $G_{c}$ the fracture energy of the material. Additionally, $\ell$ is a regularization lengthscale $(\ell>0)$ which controls the width of the transition zone between the intact and fully broken state. The parameter $k$ models an artificial (small) stiffness of the broken phase, necessary to avoid numerical difficulties (see Bourdin et al. (2000) for discussion). Such a regularized energy functional can be shown to exhibit so-called $\Gamma$-convergence as $\ell \rightarrow 0$, i.e. it converges to the original variational form of brittle 
fracture according to

$$
E\left(u_{i}, S\right)=\int_{\Omega S} \Psi\left(\epsilon_{i j}\left(u_{i}\right)\right) \mathrm{d} V+G_{c} \mathcal{H}^{N-1}(S)
$$

where $\mathcal{H}^{N-1}(S)$ denotes the Hausdorff measure of the fracture $S$ (Fig. 1), noting that the surface area is in $N=3 \mathrm{D}$ while its length is in $N=2 \mathrm{D}$. Numerically, the regularization lengthscale $\ell$ must thus be taken small enough to avoid underestimating the bulk energy. Moreover, the mesh size $h$ must be taken such that $h \ll \ell$ (independent of the order of the chosen finite element description) in order to accurately estimate the fracture energy. A criteria $h<\ell / 2$ (for quadrilateral element) appears to give a sufficient approximation of the fracture topology (Miehe et al., 2010).

The previous formulation (28) does not distinguish between traction and compression. It must therefore be modified in order to avoid non-physical fracturing in compression. This is typically done by splitting the elastic energy density into 2 additive parts related to tension $\Psi^{+}$and compression $\Psi^{-}$, respectively, and degrading only $\Psi^{+}$by $\left(s^{2}+k\right)$. Different splitting approaches are possible, either by considering the principal strain direction (Miehe et al., 2010) or via a decomposition of the strain energy in volumetric and deviatoric components (Amor et al., 2009), see Ambati et al. (2015) for a more detailed discussion. The extension to poroelastic media is possible (Miehe et al., 2015; Mikelic et al., 2015; Lee et al., 2016; Wilson and Landis, 2016), although details in the regularization differ among the various published approaches.

In order to be able to solve hydraulic fracture propagation problems using a phase field approach, two important issues need to be properly tackled due to the smearing nature of the method. The first issue relates to the pressurized nature of hydraulic fracture. Prescribing fluid pressure on the fracture surfaces can be done by replacing the integral on the crack surface in the power of external forces on the solid domain, viz.

$$
\int_{S} p\left(u_{i}^{+}-u_{i}^{-}\right) n_{i}^{-} \mathrm{d} x
$$

by a volume integral over the whole domain (cracked and uncracked). For example, Bourdin et al. (2012) perform the following regularization

$$
\int_{S} p\left(u_{i}^{+}-u_{i}^{-}\right) n_{i}^{-} \mathrm{d} x=\int_{\Omega} p u_{i} \partial_{x_{i}} s \mathrm{~d} x
$$

Using such an approximation, the plane-strain solution for the propagation of a hydraulic fracture in the toughness dominated regime (i.e. zero fluid viscosity) can 
be recovered, albeit for small enough $\ell$ and $h \approx \ell$. Another regularization has been put forward by Mikelic et al. (2015) and Lee et al. (2016) based on $\int_{\Omega} s^{2}\left(u_{i} \partial_{x_{i}} p+\right.$ $\left.p \partial_{x_{i}} u_{i}\right) \mathrm{d} x$. In essence this regularization circumvents the need to compute the exact fracture displacement jump. However, to couple lubrication flow inside the fracture with elastic deformation, the fracture width $w=\left(u_{i}^{+}-u_{i}^{-}\right) n_{i}^{-}$must be reconstructed precisely as it directly controls the non-linearity of such a coupling. Remember that Poiseuille's law (Eq. 12) is $q_{i} \propto-w^{3} \partial_{x_{i}} p$, such that accuracy on the fracture width is critical to obtain any meaningful results. This second issue is probably the most demanding for any phase field methods in the context of hydraulic fracturing.

Some further discussion on the phase field estimate of the fracture width is therefore in order. The fracture width can be approximated by $w \approx h_{e}\left(\lambda_{n}\right)$, where $h_{e}$ is the lengthscale associated with the discretization (mesh size) and $\lambda_{n}$ the nominal stretch ratio of a line element perpendicular to the fracture plane (Bourdin et al., 2012). Such an approximation appears to over-estimate the fracture width. A slightly different approach has also been used in the works by (Miehe et al., 2015; Wilson and Landis, 2016; Miehe and Mauthe, 2016), approximating $w \approx h_{e}\left(1-\lambda_{n}\right)$. This approach, combined with a proper transition between Stokes flow in the fracture and Darcy's flow in the solid (modeled as poroelastic material) using an adequate set of transition indicator functions (depending on the phase field variable $s$ ), appears to reproduce both viscosity and toughness dominated HF propagation in plane-strain (Wilson and Landis, 2016). Note that the fracture width reconstruction appears to be sensitive to the relative orientation of the fracture plan within the mesh, and requires further improvements. Lee et al. (2016) have used an integral form using the phase variable initially put forward in the context of cohesive fracture to reconstruct the displacement jump across the fracture. In a recent work, Santillán et al. (2017) have used a phase-field approach in plane-strain coupled in a staggered manner with lubrication flow in the fracture using finite volume. Although restricted to a fracture propagating along a mesh direction, this scheme appears to reproduces the different propagation regimes in plane-strain over relatively short injection duration.

It is also important to make note of the drastic mesh requirements of the phase field methods, specifically $h \ll \ell$ with $\ell$ in $O\left(10^{-4}\right)$ or less for accurate description of the fracture process. For practical purposes, mesh refinement and adaptativity together with robust parallel solvers are thus mandatory.

In summary, the smearing nature of the phase field approach is both a strength and a weakness in the context of hydraulic fracturing. It is a strength because it allows modeling of complex mixed mode fracture in 3D, fractures competition, heterogeneities, and so forth. It is a weakness because it can be challenging to satisfy the requirement for accurate reconstruction of the displacement discontinuities 
across the fracture surface. As a result, although attractive, it is still unclear how robust and accurate phase field methods can be in reproducing the whole range of hydraulic fracture propagation regime (viscosity/toughness, leak-off/storage) over a practical range of length and time scales.

\subsection{Meshless methods}

Meshless methods such as Element Free Galerkin (EFG) and Smoothed Particle Hydrodynamics (SPH) have been also used to model fracture propagation in both elastic and poroelastic materials. However, applications to hydraulic fracturing are relatively scarce and no thorough verification has been performed to date. We briefly outline these methods below.

\subsubsection{Element free galerkin}

The Element Free Galerkin (EFG) method originally developed in the 1990s (Belytschko et al., 1994) is based on nodal data only. The approximating functions in this method are usually based on moving least-square approximants. Like in XFEM, tip enrichment functions can be used to properly capture the stresses asymptotic in the near tip region. Each node is associated with a domain of influence, i.e. with a weight function which is strictly zero outside the domain of influence. In order to model fractures / discontinuities, the method requires adaptation of the weight functions of the nodes whose domain of influence is cut by the fracture. This can be done either in a sharp / discontinuous way or via a smooth approximation (Sukumar et al., 1997; Krysl and Belytschko, 1999). Ultimately, this implies to carry and

update an important data structure and perform special operations to account for fracture efficiently.

Despite its early attractiveness, the use of EFG for fracture propagation has had limited success (Sukumar et al., 1997). The reasons for its limited success are mostly related to integration procedures (underlying mesh), data structure, and weight functions for handling fracture propagation in a simple and accurate manner. Recently, Samimi and Pak (2016) have proposed a algorithm based on EFG and an implicit time stepping scheme for the propagation of a hydraulic fracture in a porous material under plane strain conditions. The fracture propagation appears to be performed in an explicit way, using a computation of the stress intensity factor to set a predefined new fracture increment. A thorough verification of the different hydraulic fracture propagation regimes is however lacking. 


\subsubsection{Smooth Particle Hydrodynamics}

Smooth Particle Hydrodynamics (SPH) is a Lagrangian particle based method whose development was initially geared toward complex fluid dynamics problems (see the reviews of Monaghan $(1992,2005)$ ). The extension to elasticity and fracture dates back to the 1990s (Libersky and Petschek, 1991; Benz and Asphaug, 1995) focusing mostly on fragmentation problem. It has been used to address some geological problems (Gray and Monaghan, 2004; Das and Cleary, 2010). Applications to hydraulic fracturing (Douillet-Grellier et al., 2016) are preliminary in nature, i.e. they are limited to uniform pressure cases for which the fluid coupling vanishes except for the global volume balance. As a result it remains unclear how well SPH is suited for the solution of quasi-static hydraulic fracturing problems.

\section{Meso-scale models}

The use of particle assemblies to represent solid deformation, the so-called Distinct Element Method (DEM), can be traced back to the early work of Cundall (1971). In the DEM, the mechanical behaviors are realized through the displacement of the particles potentially with different sizes and shapes and the fractures are represented in the states of the contacts between the particles. Later on, the lattice model with point-like mass and small-displacement (including rotation) spring was used to model solid deformation. The configuration is simplified from the bonded particle assembly to the bond only. The stress and deformation of the springs are connected by some constitutive laws that should be related to some deformation mechanisms (Grassl et al., 2015).

In addition to mechanical responses, the fluid flow in these meso-scale models is realized by fluid transport elements, another mesh different from the mechanical mesh, represented by pipelines connecting the broken contacts in particle-based models (Damjanac et al., 2013, 2015), or/and by conduits along the edge of the Voronoi cells in lattice models (Grassl et al., 2015). Coupling with a finite volume scheme has also been proposed (Papachristos et al., 2017) . Depending on the formulation, some idealizations are performed to model fluid flow, for example, the use of steady-state solution of fluid pressure in a saturated porous medium (Grassl et al., 2015).

Recently, a nonlocal description of the bonds or springs was used to simulate elastic deformation and fracture growth within the framework of peridynamics (Silling, 2000). In this model, the concept of stress is replaced by pair of forces in construct- 
ing the equation of equilibrium for a node. An integral form of all interacting forces in a given domain (horizon) is produced and the long-range forces are included in contrast to the local, discrete models like DEM or quasi-random lattice model. This numerical technique has been coupled to fluid flow (Ouchi et al., 2015). In that work, the critical energy density stored in a bond is used for material failure and the flow in the fracture is described as a consolidation problem. Comparisons with the plane-strain hydraulic fracture solution were performed with a reasonable agreement on the fracture length only at early time.

Only a few of these meso-scale models have been tested against known solutions for the propagation of a planar radial hydraulic fracture (Damjanac et al., 2015; Lecampion et al., 2013). They appear to be able to reproduce these known solutions, albeit at a very large computation cost and with these benchmarking exercises limited to only specific combinations of model resolution, size, and initial stresses.

Besides large computational time, the main difficulty of these type of models lies in the calibration of the micro constitutive parameters. This task can be partly automated as shown by Damjanac and Cundall (2016). A particularly important point relates to the macroscopic fracture energy. Macroscopic fracture toughness is intrinsically related to the meso-scale discretization lengthscale (Huang and Detournay, 2008; Huang et al., 2013); it fixes the meso-scopic discretization length of this type of model. These meso-scale models allow to investigate with little additional developments very complex fracture geometries, such as near-wellbore effects on fracture growth (Grassl et al., 2015) and interactions with pre-existing fractures in 3D (Damjanac and Cundall, 2016). To save computational time, a hybrid DEM and continuum method with a discrete inner region embedded in an outer continuum domain has recently been developed to solve the $2 \mathrm{D}$ problems with complex fracture geometries (Zhang et al., 2017a). In this paper, the authors attempted to verify their results with plane-strain analytic solutions.

\section{$6 \quad$ Engineering models}

By engineering models, we refer to models that make some further simplifications within the basic model presented in section 2.1 in order to significantly decrease computational cost, thus enabling practical engineering design. often these underlying simplifications are guitable for particular configuration(s). These models are often fit for certain purposes and as such their limitations must therefore be properly understood, especially because the various models can vary significantly among each other both in underlying simplifications and in solutions even to relatively sim- 
ple hydraulic fracturing problems. Some appreciation of the diversity of modeling approaches can be gained from the classical comparative study of Warpinski et al. (1993). In this review, after a brief description of the underlying simplifications and limitations, our focus will be on some recent advances without attempting to comprehensively review many various models and their specific approaches to simplification and generalization.

The Pseudo-3D (P3D) models (Simonson et al., 1978), as an extension of the well-known PKN (Perkins-Kern-Nordgren) model (Perkins and Kern, 1961; Nordgren, 1972; Kemp, 1990), divides the fracture into cells along the lateral direction, the width and height of which are calculated solely based on local fluid pressure. P3D models can handle the fracture height growth, and maintain the lateral coupling for fluid pressure, so that the initial 3D problem is reduced to a $2 \mathrm{D}$ elastic problem and a 1D fluid flow problem. The dimension reduction can significantly reduce computational time. However, the original formulation of P3D models did not consider the elastic coupling along the lateral direction and the effect of the fracture toughness.

This drawback of the classical PKN model, which requires the fluid pressure to be zero at the fracture tip, has been long recognized. Meyer (1989), for example, addressed this issue by essentially assuming a semi-circular leading edge and computing the stress intensity factor accordingly, making use of an influence function both on the stress intensity factor and on the elasticity equation itself, in order to ensure that the model captures in an approximated way the limits of a circular and finger-like (PKN) hydraulic fracture. More recent remedies rely on a near-tip displacement formulation derived from asymptotic analysis, as a measure for fracture growth, which has been developed by Adachi et al. (2010) for a similarity solution if the fracture front is flat and by Kovalyshen and Detournay (2009) for a finger-like fracture. These latter approaches draw on a formal consideration of the non-local elasticity for a finger-like fracture that was introduced by Adachi and Peirce (2008).

Related to the resolution of the near-tip displacement field, some recent approaches have been introduced to account for fracture propagation. These include and energy approach based on near-tip nonlocal elasticity (Dontsov and Peirce, 2015a; Sarvaramini and Garagash, 2015). Moreover, the PKN and P3D models have been recently extended to include both the non-local elastic interactions between the cells and the near-tip displacement formulation (Dontsov and Peirce, 2015a, 2016). This modification increases the overall accuracy of the model in fracture opening, height and length, which especially affects toughness dominated cases. It should be noted that in addition to accurately describing elastic deformation, further refinement can be obtained by replacing the 1D fluid flow along the lateral direction by 
2D fluid flow within the vertical fracture surface, as done by Weng (1992). This inclusion of 2D fluid flow is sometimes essential for restricting unstable height growth based on the uniform pressure assumption.

The cell-based P3D model is also the foundation for extensions that consider growth of hydraulic fractures in formations with many pre-defined natural fractures (e.g. Weng et al., 2011a; Meyer and Bazan, 2011). When the fracture height growth is involved during the fracture network formation, the stacked cell model consisting of many rows of elements in the vertical direction was proposed by Cohen et al. (2015). The stress and fluid pressure profiles vary vertically and the fracture height prediction becomes more accurate than the conventional P3D models if the in-situ stress and material property differences cannot be neglected (Zhang et al., 2017b). Moreover, for multiple fracture growth in a single layer along the horizontal well, a 3D correction factor has been used to extend bi-dimensional plane-strain model to the 3D case (Wu and Olson, 2015b,a). Such a dimension reduction model enforces the fracture(s) height to be constant and equal to a pre-defined value equal to the layer thickness. While this assumption may be somewhat harsh and they reduce the practicality of the model from the perspective of eliminating its ability to predict fracture containment, the benefits lay in the possibility to simulate the propagation of a large number of hydraulic fractures and their interactions with vertical preexisting ones at a reasonable computational cost.

It is important to note that other approaches to simulate hydraulic fractures in naturally fractured formations are available using a 2D (e.g. Olson and DahiTaleghani, 2009) or 3D Displacement Discontinuity Method (e.g. McClure and Horne, 2013), or using an adaptation of the Distinct Element Method (e.g. Damjanac et al., 2010; Nagel et al., 2011). The latter one sacrifices computational time for a more detailed and/or general description of the mechanics problem. On the other hand, P3D-based are aimed at practical design. All of these models are aimed at addressing one of the major challenges of modern hydraulic fracture simulation, which is to predict whether a given stimulation will result in a simple geometry with only one or a few dominant, somewhat planar fracture branches or a multiply-branched "complex" geometry. A recent reviews of field evidence, modeling insights, and laboratory results is given by Bunger and Lecampion (2017). Also, for all of these models, one of the greatest challenges lies in appropriately defining the conditions under which a hydraulic fracture will cross each natural fracture it encounters. Because this issue is essentially one of physics and not of numerical simulation per se, we will not attempt to review the large relevant literature but will instead refer to the review provided by Chuprakov et al. (2014). Suffice it to say, however, that regardless of the simulation method, this choice of crossing condition may have a 
predominant impact on the numerical predictions (Kresse et al., 2013).

\section{The necessity of verification and validation}

Verification (checking that a model indeed solves what it claims to solve) and validation (checking that a model hypotheses are indeed acceptable) of numerical models are both essential. This is particularly needed for the simulation of hydraulic fractures. Since it is a non-linear moving boundary problem, numerical errors may accumulate in time resulting in widely different results between codes after the simulation of an hour of injection (Warpinski et al., 1994). Fortunately, over the last two decades, a number of semi-analytical solutions for simple planar fracture geometries (plane-strain, axi-symmetric fracture) have been obtained (see Detournay, 2016, 2004, for a review). Any numerical models must thus be verified against these analytical solutions before tackling more complex configurations for which analytical solutions are unavailable. Nonetheless, it remains that a large number of authors either do not perform any comparisons with these solutions, or actually use old and incomplete solutions such as Geertsma and De Klerk (1969) for the plane strain hydraulic fracture.

In Table 1, we list the known semi-analytical solutions valid in the different propagation regimes for three simple fracture geometries (plane-strain, radial and height contained). The proper verification of a simulator must be performed on all these different limiting propagation regimes (storage / leak-off, viscosity / toughness). In addition to these, complete analytical approximations have also been developed recently for these two geometries (Dontsov, 2016, 2017). Some semi-analytical solutions for power-law fluids also exist (Adachi and Detournay, 2002; Garagash, 2006a). Transient numerical solutions also exist for the case of a fluid lag (Lecampion and Detournay, 2007; Gordeliy and Detournay, 2010). For model verification, the evolution of fracture length versus time (for a sufficiently large time of injection spanning at least two decades of time) as well as the fracture opening and net pressure profiles at different times must be compared to analytic solutions, and relative errors computed. From our experience, it is relatively common that a scheme giving an accurate solution in a given limiting regime (e.g. toughness/storage dominated), may perform poorly in another (e.g. viscosity/storage dominated).

The comparisons with analytical solutions also allows to better understand the benefits and constraints of different numerical schemes. Figure 4 shows an example of such an exercise where the convergence to the plane-strain self-similar viscosity dominated propagation solution was investigated for different numerical models 


\begin{tabular}{|l|}
\hline Known analytical solutions for HF propagation \\
\hline Plane-strain hydraulic fracture (KGD) \\
\hline \hline Storage (impermeable) / large toughness and small viscosity correction (Garagash, 2006b) \\
Storage/ large viscosity (Adachi and Detournay, 2002; Spence and Sharp, 1985) \\
Storage /large viscosity small toughness correction (Garagash and Detournay, 2005) \\
Large toughness with small or large leak-off (Bunger et al., 2005a) \\
Large viscosity with small or large leak-off (Adachi and Detournay, 2008) \\
Storage (impermeable) with a fluid lag: early time solution (Garagash, 2006c) \\
\hline Radial hydraulic fracture (penny-shaped) \\
\hline \hline Storage / Large toughness (Abé et al., 1976) \\
Storage / Large viscosity (and small toughness correction) (Savitski and Detournay, 2002) \\
Large toughness with small or large leak-off (Bunger et al., 2005a) \\
Leak-off / Viscosity solutions (Madyarova, 2003) \\
Impermeable with fluid lag: early time solution (Bunger and Detournay, 2007) \\
\hline Height contained hydraulic fracture (PKN) \\
\hline \hline Large viscosity with or without leak-off (Kemp, 1990; Kovalyshen and Detournay, 2009) \\
\hline Inclusion of toughness (Dontsov and Peirce, 2016; Sarvaramini and Garagash, 2015) \\
\hline
\end{tabular}

Table 1: Analytical or semi-analytical (via truncated series) solutions of hydraulic fracture propagation in limiting regimes for simple fracture geometries. Large toughness implies zero viscosity, similarly large viscosity implies zero toughness. Solutions for small or large toughness also exist. Impermeable versus porous cases are related to the storage and leak-off dominated regimes. These different limiting solutions are to be understood in the light of a proper scaling analysis (see the reviews (Detournay, 2004, 2016), and discussions in the papers cited in this table). Some complete solutions (storage / leak off / toughness/ viscosity) - although numerical- exist for the plane-strain (Hu and Garagash, 2010) and radial geometries (Madyarova, 2003). 
based on different propagation criteria (LEFM, cohesive zone models, HF tip asymptotics, and so forth). Some models performed better than others. Through such an exercise, the trade off between accuracy, computational efficiency, and robustness (the ability to model more complex configuration without fine-tuning) can be clearly demonstrated.

Besides analytical/semi-analytical solutions, carefully designed laboratory experiments can also provide important benchmarks for numerical simulators. A number of laboratory scale hydraulic fracture experiments have been performed with detailed measurements of the evolution of the fracture front, fracture width profiles, and the wellbore-pressure. Design of the experiments relies on scaling of the physical quantities (specimen dimensions, injection rate, material properties) so that the laboratory scale hydraulic fractures are dominated by similar physical processes to field scale hydraulic fractures (e.g. Bunger et al., 2005c; Bunger, 2008; Bunger and Detournay, 2008). While scaling and experimental design can be challenging, especially for issues such as proppant transport and hydraulic fracture growth in media with heterogeneities at a wide range of scales (like most rocks), there are a number of recent examples that highlight the lessons that can be learned from benchmarking simulators with experiments. Furthermore, in many cases experiments enable benchmarking in configurations for which no analytical solution is possible. While not attempting to review the substantial experimental hydraulic fracturing literature (a broader recent review is available in Bunger and Lecampion (2017)), we present here some illustrative examples mainly involving the present authors' work, thus enabling some commentary on overarching conclusions which may not be evident from the original works along. These selected examples include:

- Experiments on height growth in stress layered media, which were used to validate the basic Planar 3D approach to height growth prediction (Wu et al., 2008; Jeffrey and Bunger, 2009). One of the striking findings in the course of this work is that it is challenging to predict wellbore pressure even when the simulator is matching well with the evolution of the hydraulic fracture front and width. These experimental data have recently been extended to include the influence of weak bedding planes by Xing et al. (In Review).

- Experiments demonstrating the theoretically-predicted multiscale nature of the near tip region of hydraulic fractures (Bunger and Detournay, 2008; Xing et al., 2017). These experiments confirm that the steady-state traveling wave type solution (see Section 2.1.6) successfully predicts the family of near tip solutions and each solution's emergence at the observable scale in different propagation regimes. Also, the experiments show that the predicted asymptotic 


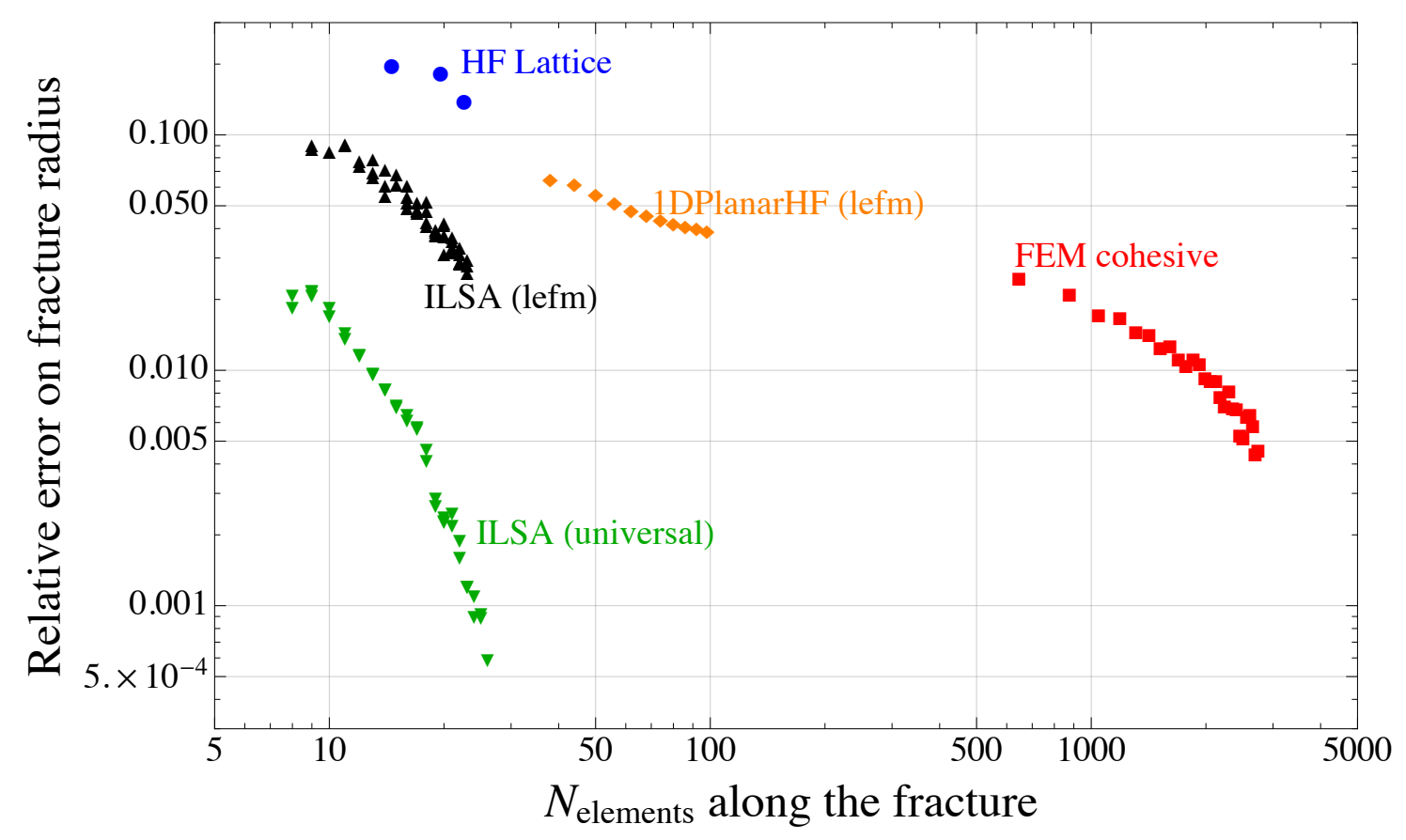

Figure 4: Relative error on hydraulic fracture radius of different numerical schemes as function of the number of elements discretizing the fracture. The results are for the early-time storage/viscosity dominated regime where they can be compared to the semi-analytical solution of Savitski and Detournay (2002) in that propagation regime. All the simulations were performed with a regular mesh. The different schemes reach the same accuracy at different numerical cost (i.e. different number of elements). The implicit level set algorithm (ILSA) using the complete (universal) hydraulic fracture asymptote (see sub-section 4.1) can achieve very accurate solution on very coarse mesh, more elements (about 10 times more) are needed when using the linear elastic fracture mechanics asymptote. A finite element scheme with a cohesive zone model reaches the same accuracy with a much larger number of elements (about 200 times more elements). Similarly, the meso-scale HF-lattice based simulation is much less accurate at the same level of discretization. Figure adapted from Lecampion et al. (2013). 
forms emerge even for curving hydraulic fractures and those with substantial fluid lag (Bunger et al., 2005b), which is important for numerical simulations but yet unproven theoretically.

- Experiments showing growth of curving hydraulic fractures in the presence of a nearby free surface (Bunger et al., 2004; Bunger, 2005; Bunger et al., 2008) and/or in the vicinity of a nearby, previously-placed hydraulic fracture (Bunger et al., 2011). Numerical simulations have been shown to capture the behavior of these experiments (Bunger et al., 2013), with the notable observation that hydraulic fractures curve towards a free surface in all cases but can curve towards or away from previously-placed hydraulic fractures depending on the magnitude of the minimum confining stress. Note that the effect of the early stage transient injection rate was also found to be important and a subsequent study comparing to a wider range of experiments also found that capturing this early transient behavior is essential to matching laboratory scale hydraulic fractures (Lecampion et al., 2017).

The necessity of benchmarking hydraulic fracture propagation codes with both analytical solutions and laboratory experiments is compounded by the fact that field data are often insufficient to carry out a proper validation of a numerical code. Even in the best possible case, where many measurements are performed and a mineback of the hydraulic fracture performed (e.g. Warpinski and Teufel, 1987; Jeffrey et al., 2009), a degree of uncertainty on the response of the rock always remain as measurements are always too scarce spatially. If the response of a field experiment can not be reproduced by a numerical model, ones needs to have sufficient confidence in the capabilities of the numerical model to be able to decipher between a missing feature in the model (heterogeneity, other physical process not included) and poor numerical performance. This can only be achieved if the numerical code has been thoroughly tested beforehand.

\section{Conclusions}

Accelerating development of numerical methods for hydraulic fracture simulation in the past two decades has led to both enhancements of classical methods as well as methods that, while imported from other fields of mechanics, are completely new in their application to hydraulic fracturing. Examples of enhancements of classical methods include boundary element-based approaches that are enriched to recog-

nize the multi-scale nature of a propagation hydraulic fracture tip thereby enabling 
accurate simulation on very coarse meshes. Examples of completely new methods to hydraulic fracturing include Smoothed Particle Hydrodynamics and Phase Field methods among others. All these methods and the different fracture propagation scheme have their benefits and pitfalls. Their suitability (and accuracy) depends on the purpose of the modeling and the specific types of hydraulic fracture simulation to be undertaken. None provides a universal, "off the shelf" solution to all hydraulic fracturing problems.

In reviewing the different numerical techniques, we observe that some models are presented with verification and validation, showing how even complex models reduce in given limits to benchmark solutions or match in certain cases to laboratory experiments. However, as the draw of "complexity" becomes ever stronger, there is also an increasing tendency in the literature to present new models in terms of their added features and ability to illustrate complicated behaviors which could occur, but without verification and validation. Such a trend will certainly prove increasingly problematic as we fall into the pitfalls forecasted three decades ago by Starfield and Cundall (1988) in which we:

"...have an implicit credo that more detail implies a better model. It is an additive credo: the modeller becomes hooked on bigger and "better" models and these in turn need more data, leading to more field and laboratory measurements. At best these efforts are a waste of time and resources; at worst they are counter-productive, concealing the [forest] for the trees. After all, we build models because the real world is too complex for our understanding; it does not help if we build models that are also too complex. The art of modelling lies in determining what aspects of the geology are essential for the model."

The process of verification and validation, in the words of Starfield and Cundall (1988), seeking to "gain confidence in the model", is critical for avoiding such pitfalls, ensuring understanding of the basic elements of each model and enabling users making informed choices among different numerical schemes (see Fig. 4).

A large number of challenges remain in relation to the increasing demand for high resolution modeling of ever more complex configurations, including:

- The still-unresolved 3D fracture propagation criteria under truly mixed mode condition with the associated front segmentation and curving is seldom recognized and addressed in the context of hydraulic fracture modeling.

- The possibility of predicting the micro-seismic activity associated with fracturing has received a lot of interest as it is often the only available data in practice. However, most predictions are currently based on models which do 
not account for the details of the physical processes associated with these small scale dynamic ruptures. How good are those predictions is yet unclear.

- The details of the interaction between pre-existing fractures and a growing hydraulic fractures is not completely resolved, especially when accounting for the three dimensional nature of such interactions. Here again, more laboratory experiments are clearly required to decipher between different modeling approaches.

- The modeling of simultaneous hydraulic fracture propagation from a single wellbore requires the coupling between multiple fracture growth and wellbore flow dynamics in order to determine the fluid rates entering each of the propagating fractures. The details are often overlook and introduce a set of additional non-linearities.

- The non-linearities of the fracturing fluid (complex shear-thinning behavior, viscoelasticity) require an improved description beyond the simple power-law model, not to mention the slurry rheology of the fracturing fluid/proppant suspensions. Similarly, rock non-linearities (plasticity, damage) needs to be accounted for in some cases.

Finally, one should always keep in mind that a large number of other features must be included in order to perform an engineering design of a hydraulic fracturing treatment. Notably, the temperature dependence of the rheology of fracturing fluids, the details of proppant transport, fluid scheduling, the mechanics and physics of fracture closure after shut-in, the degradation of fracturing fluid viscosity post placement among others must all be properly modeled in any industrial software for hydraulic fracturing engineering.

In summary, the acceleration of hydraulic fracture simulation in the past two decades has led to astounding advances. A number of challenges remain and are amplified with a pressing drive toward fully-coupled, three-dimensional hydraulic fracture modeling that accounts for host-rock heterogeneity. In the context of such a drive to complex models, we argue that the importance of best-practice development that includes careful verification and validation is vital to ensure that progress is constrained by the appropriate underlying physics and mathematics with a constant attention to identifying conditions under which simpler models suffice for the intended modeling purposes. 
Numerical methods for hydraulic fracture propagation

Lecampion et al.

\section{References}

H. Abé, L. M. Keer, and T. Mura. Growth rate of a penny-shaped crack in hydraulic fracturing offset rocks. Journal of Geophysical Research, 81:6292-6298, 1976. doi: 10.1029/JB081i035p06292.

J. I. Adachi and E. Detournay. Self-similar solution of a plane-strain fracture driven by a power-law fluid. International Journal for Numerical and Analytical Methods in Geomechanics, 26(6):579-604, 2002. ISSN 0363-9061. doi: 10.1002/nag.213.

J. I. Adachi and E. Detournay. Plane strain propagation of a hydraulic fracture in a permeable rock. Engineering Fracture Mechanics, 75(16):4666-4694, 2008. ISSN 00137944. doi: 10.1016/j.engfracmech.2008.04.006.

J. I. Adachi and A. P. Peirce. Asymptotic analysis of an elasticity equation for a finger-like hydraulic fracture. Journal of Elasticity, 90(1):43-69, 2008.

J. I. Adachi, E. Siebrits, A. P. Peirce, and J. Desroches. Computer simulation of hydraulic fractures. International Journal of Rock Mechanics and Mining Sciences, 44(5):739-757, 2007. ISSN 13651609. doi: 10.1016/j.ijrmms.2006.11.006.

J. I. Adachi, E. Detournay, and A. P. Peirce. Analysis of the classical pseudo-3D model for hydraulic fracture with equilibrium height growth across stress barriers. International Journal of Rock Mechanics and Mining Sciences, 47:625-639, 2010.

S. H. Advani, T. S. Lee, and J. K. Lee. Three-dimensional modeling of hydraulic fractures in layered media: part I- finite element formulations. Journal of Energy Resources Technology, 112(1):1-9, 1990.

M. Ambati, T. Gerasimov, and L. De Lorenzis. A review on phase-field models of brittle fracture and a new fast hybrid formulation. Computational Mechanics, 55 (2):383-405, 2015.

H. Amor, J. J. Marigo, and C. Maurini. Regularized formulation of the variational brittle fracture with unilateral contact: Numerical experiments. Journal of the Mechanics and Physics of Solids, 57(8):1209-1229, 2009.

A. C. Barbati, J. Desroches, A. Robisson, and G. H. McKinley. Complex fluids and hydraulic fracturing. Annual Review of Chemical and Biomolecular Engineering, 7:415-453, 2016. 
Numerical methods for hydraulic fracture propagation

Lecampion et al.

G. I. Barenblatt. The mathematical theory of equilibrium cracks in brittle fracture. Advances in Applied Mechanics, VII:55-129, 1962.

R. D. Barree and H. Mukherjee. Determination of pressure dependent leakoff and its effect on fracture geometry. In Proceedings SPE Annual Technical Conference and Exhibition, Denver, Colorado, USA, October 6-9 1996. SPE 36424.

T. Belytschko and T. Black. Elastic crack growth in finite elements with minimal remeshing. International Journal for Numerical Methods in Engineering, 45:601620, 1999.

T. Belytschko, Y. Y. Lu, and L. Gu. Element-free galerkin methods. International Journal for Numerical Methods in Engineering, 37(2):229-256, 1994.

T. Belytschko, R. Gracie, and G. Ventura. A review of extended/generalized finite element methods for material modeling. Modelling and Simulation in Materials Science and Engineering, 17(4):043001, 2009.

W. Benz and E. Asphaug. Simulations of brittle solids using smooth particle hydrodynamics. Computer Physics Communications, 87(1-2):253-265, 1995.

M. Bonnet. Boundary integral equation methods for solids and fluids. John Wiley \& Sons, 1999.

M. Bonnet, G. Maier, and C. Polizzotto. Symmetric galerkin boundary element methods. Applied Mechanics Reviews, 51:669-704, 1998.

T. J. Boone and A. R. Ingraffea. A numerical procedure for simulation of hydraulically-driven fracture propagation in poroelastic media. International Journal for Numerical and Analytical Methods in Geomechanics, 14(1):27-47, 1990 .

B. Bourdin, G. A. Francfort, and J. J. Marigo. Numerical experiments in revisited brittle fracture. Journal of the Mechanics and Physics of Solids, 48(4):797-826, 2000 .

B. Bourdin, C. P. Chukwudozie, and K. Yoshioka. A variational approach to the numerical simulation of hydraulic fracturing. In SPE Annual Technical Conference and Exhibition. Society of Petroleum Engineers, 2012.

A. P. Bunger. Near-surface hydraulic fracture. PhD thesis, University of Minnesota, 2005. 
A. P. Bunger. A rigorous tool for evaluating the importance of viscous dissipation in sill formation: it's in the tip. In C. Annen and G. Zellmer, editors, Dynamics of Crustal Magma Transfer, Storage, and Differentiation, volume 304, pages 71-81. Geological Society, London, 2008.

A. P. Bunger and E. Detournay. Early-time solution for a radial hydraulic fracture. Journal of engineering mechanics, 133(5):534-540, 2007.

A. P. Bunger and E. Detournay. Experimental validation of the tip asymptotics for a fluid-driven crack. Journal of the Mechanics and Physics of Solids, 56:3101-3115, 2008. doi: $10.1016 /$ j.jmps.2008.08.006.

A. P. Bunger and B. Lecampion. Four critical issues for sucessful hydraulic fracturing applications. In to appear in Comprehensive Rock Engineering, CRC Press, 2017.

A. P. Bunger and A. P. Peirce. Numerical simulation of simultaneous growth of multiple interacting hydraulic fractures from horizontal wells. In ASCE Shale Energy Engineering Conference, Pittsburgh, PA, 2014. ASCE.

A. P. Bunger, R. G. Jeffrey, and E. Detournay. Toughness-dominated near-surface hydraulic fracture experiments. In D. Yale, S. Willson, and A. Abou-Sayed, editors, Gulf Rocks 2004 - Proc 6th North American Rock Mechanics Symposium, Houston, 2004. Paper No 468.

A. P. Bunger, E. Detournay, and D. I. Garagash. Toughness-dominated hydraulic fracture with leak-off. International Journal of Fracture, 134(2):175190, 2005a. ISSN 0376-9429. doi: 10.1007/s10704-005-0154-0. URL http: //www.springerlink.com/index/10.1007/s10704-005-0154-0.

A. P. Bunger, E. Detournay, and R. G. Jeffrey. Crack tip behavior in near-surface fluid-driven fracture experiments. C. R. Mècanique, 333:299-304, 2005b.

A. P. Bunger, R. G. Jeffrey, and E. Detournay. Application of scaling laws to laboratory-scale hydraulic fractures. In G. Chen, S. Huang, W. Zhou, and J. Tinucci, editors, Alaska Rocks 2005 - Proc 40th U.S. Rock Mechanics Symposium, Anchorage, 2005c. Paper No 818.

A. P. Bunger, R. G. Jeffrey, and E. Detournay. Evolution and morphology of saucershaped sills in analog experiments. In K. Thompson and N. Petford, editors, Structure and Emplacement of High-Level Magmatic Systems, volume 302, pages 107-118. Geological Society, London, 2008. 
A. P. Bunger, R. G. Jeffrey, and X. Zhang. Experimental investigation of the interaction among closely spaced hydraulic fractures. In Proceedings 45th U.S. Rock Mechanics Symposium, San Francisco, CA, USA, 2011. Paper No. 11-318.

A. P. Bunger, E. Gordeliy, and E. Detournay. Comparison between laboratory experiments and coupled simulations of saucer-shaped hydraulic fractures in homogeneous brittle-elastic solids. Journal of the Mechanics and Physics of Solids, 61(7):1636-1654, 2013.

P. J. Carreau. Rheological equations from molecular network theories. Transactions of the Society of Rheology, 16(1):99-127, 1972.

B. Carrier and S. Granet. Numerical modeling of hydraulic fracture problem in permeable medium using cohesive zone model. Engineering Fracture Mechanics, 79:312-328, 2012.

B. J. Carter, J. Desroches, A. R. Ingraffea, and P. A. Wawrzynek. Simulating fully 3D hydraulic fracturing. Modeling in Geomechanics, 200:525-557, 2000.

J. L. Castillo. Modified fracture pressure decline analysis including pressuredependent leakoff. In Proceedings SPE Low Permeability Reservoirs Symposium, pages 273-281, Denver, Colorado, USA, 1987. SPE 16417.

E. Chekhonin and K. Levonyan. Hydraulic fracture propagation in highly permeable formations, with applications to tip screenout. International Journal of Rock Mechanics and Mining Sciences, 50:19-28, 2012.

C. H. Chen, T. Cambonie, V. Lazarus, M. Nicoli, A. J. Pons, and A. Karma. Crack front segmentation and facet coarsening in mixed-mode fracture. Physical Review Letters, 115(26):265503, 2015.

Z. Chen, A. P. Bunger, X. Zhang, and R. G. Jeffrey. Cohesive zone finite elementbased modeling of hydraulic fractures. Acta Mechanica Solida Sinica, 22(5):443452, 2009. ISSN 0894-9166. doi: 10.1016/S0894-9166(09)60295-0. URL http: //linkinghub.elsevier.com/retrieve/pii/S0894916609602950.

Z. R. Chen. Finite element modelling of viscosity-dominated hydraulic fractures. Journal of Petroleum Science and Engineering, 88-89:136-144, 2012.

S. Cherny, V. Lapin, D. Esipov, D. Kuranakov, A. Avdyushenko, A. Lyutov, and P. Karnakov. Simulating fully 3D non-planar evolution of hydraulic fractures. International Journal of Fracture, pages 1-31, 2016. 
D. A. Chuprakov, O. Melchaeva, and R. Prioul. Injection-sensitive mechanics of hydraulic fracture interaction with discontinuities. Rock Mechanics and Rock Engineering, 47(5):1625-1640, 2014.

C. E. Cohen, O. Kresse, and X. Weng. A new stacked height growth model for hydraulic fracturing simulation. In 49th US Rock Mechanics/Geomechanics Symposium, number ARMA 15-73, San Francisco, CA, USA, 2015. Americal Rock Mechanics Association.

M. L. Cooke and D. D. Pollard. Fracture propagation paths under mixed mode loading within rectangular blocks of polymethyl methacrylate. Journal of Geophysical Research: Solid Earth, 101(B2):3387-3400, 1996.

M. M. Cross. Rheology of non-Newtonian fluids: a new flow equation for pseudoplastic systems. Journal of Colloid Science, 20(5):417-437, 1965.

S. L. Crouch and A. M. Starfield. Boundary element methods in solid mechanics. George Allen \& Unwin, 1983.

P. A. Cundall. A computer model for simulating progressive large-scale movement in blocky rock system. volume 1, Nancy, France, 1971.

B. Damjanac and P. A. Cundall. Application of distinict element methods to simulation of hydraulic fracturing in naturally fractured reservoirs. Computer and Geotechnics, 71:293-294, 2016.

B. Damjanac, I. Gil, M. Pierce, M. Sanchez, A. van As, and J. McLennan. A new approach to hydraulic fracturing modeling in naturally fractured reservoirs. In Proceedings 44th U.S. Rock Mechanics Symposium, Salt Lake City, Utah, USA, June 27-30 2010. ARMA 10-400.

B. Damjanac, C. Detournay, and P. A. Cundall. Three-dimensional numerical model of hydraulic fracturing in fractured rock masses. In A. P. Bunger, R. G. Jeffrey, and J. McLennan, editors, Effective and Sustainable Hydraulic Fracturing, Brisbane, Australia, 2013. Intech.

B. Damjanac, C. Detournay, and P. A. Cundall. Application of particle and lattice codes to simulation of hydraulic fracturing. Computational Particle Mechanics, pages 1-13, 2015. 
R. Das and P. W. Cleary. Effect of rock shapes on brittle fracture using smoothed particle hydrodynamics. Theoretical and Applied Fracture Mechanics, 53(1):4760, 2010.

J. Desroches and M. Thiercelin. Modelling the propagation and closure of microhydraulic fractures. International Journal of Rock Mechanics and Mining Sciences \&f Geomechanics Abstracts, 30(7):1231-1234, 1993.

J. Desroches, E. Detournay, B. Lenoach, P. Papanastasiou, J. R. A. Pearson, M. Thiercelin, and A. Cheng. The crack tip region in hydraulic fracturing. Proceedings of the Royal Society of London. Series A: Mathematical and Physical Sciences, 447(1929):39, 1994. ISSN 1364-5021. URL http://rspa. royalsocietypublishing.org/content/447/1929/39. short.

E. Detournay. Propagation regimes of fluid-driven fractures in impermeable rocks. International Journal of Geomechanics, 4(1):35, 2004. ISSN 15323641. doi: 10. 1061/(ASCE)1532-3641(2004)4:1(35).

E. Detournay. Mechanics of hydraulic fractures. Annual Review of Fluid Mechanics, 48:311-339, 2016.

E. Detournay and D. I. Garagash. The near-tip region of a fluid-driven fracture propagating in a permeable elastic solid. Journal of Fluid Mechanics, 494:1-32, 2003. ISSN 00221120. doi: 10.1017/S0022112003005275. URL http://www. journals.cambridge.org/abstract_S0022112003005275.

E. Detournay and A. P. Peirce. On the moving boundary conditions for a hydraulic fracture. International Journal of Engineering Science, 84:147-155, 2014.

E. V. Dontsov. An approximate solution for a penny-shaped hydraulic fracture that accounts for fracture toughness, fluid viscosity and leak-off. Royal Society Open Science, 3(12):160737, 2016.

E. V. Dontsov. An approximate solution for a plane strain hydraulic fracture that accounts for fracture toughness, fluid viscosity, and leak-off. International Journal of Fracture, pages 1-17, 2017.

E. V. Dontsov and A. P. Peirce. An enhanced pseudo-3D model for hydraulic fracturing accounting for viscous height growth, non-local elasticity, and lateral toughness. Engineering Fracture Mechanics, 142:116-139, 2015a. 
E. V. Dontsov and A. P. Peirce. Comparison of toughness propagation criteria for blade-like and pseudo-3D hydraulic fractures. Engineering Fracture Mechanics, 160:238-247, 2016.

E.V. Dontsov and A. P. Peirce. A lagrangian approach to modelling proppant transport with tip screen-out in KGD hydraulic fractures. Rock Mechanics and Rock Engineering, 48(6):2541-2550, 2015b.

E.V. Dontsov and A. P. Peirce. A multiscale implicit level set algorithm (ILSA) to model hydraulic fracture propagation incorporating combined viscous, toughness, and leak-off asymptotics. Computer Methods in Applied Mechanics and Engineering, 313:53-84, 2017.

T. Douillet-Grellier, R. Pramanik, K. Pan, A. Albaiz, B. D. Jones, H. Pourpak, J. R. Williams, et al. Mesh-free numerical simulation of pressure-driven fractures in brittle rocks. In SPE Hydraulic Fracturing Technology Conference, number SPE 179138. Society of Petroleum Engineers, 2016.

D. S. Dugdale. Yielding of steel sheets containing slits. Journal of the Mechanics and Physics of Solids, 8(2):100-104, 1960.

M. J. Economides and K. G. Nolte. Reservoir Stimulation. John Wiley \& Sons, 2000.

F. Erdogan and G. C. Sih. On the crack extension in plates under plane loading and transverse shear. Journal of Basic Engineering, 85(4):519-527, 1963.

M. Faivre, B. Paul, F. Golfier, R. Giot, P. Massin, and D. Colombo. 2D coupled HM-XFEM modeling with cohesive zone model and applications to fluid-driven fracture network. Engineering Fracture Mechanics, 159:115-143, 2016.

G. A. Francfort and J. J. Marigo. Revisiting brittle fracture as an energy minimization problem. Journal of the Mechanics and Physics of Solids, 46(8):1319-1342, 1998.

T. P. Fries and T. Belytschko. The extended/generalized finite element method: an overview of the method and its applications. International Journal for Numerical Methods in Engineering, 84(3):253-304, 2010.

P. Fu, S.M. Johnson, and C.R. Carrigan. An explicitly coupled hydro-geomechanical model for simulating hydraulic fracturing in arbitrary discrete fracture networks. 
International Journal for Numerical and Analytical Methods in Geomechanics, 37 (14):2278-2300, 2013. doi: 10.1002/nag.2135.

D. I. Garagash. Transient solution for a plane-strain fracture driven by a shearthinning, power-law fluid. International Journal for Numerical and Analytical Methods in Geomechanics, 30(14):1439-1475, 2006a. doi: 10.1002/nag.

D. I. Garagash. Plane-strain propagation of a fluid-driven fracture during injection and shut-in: asymptotics of large toughness. Engineering Fracture Mechanics, 73 (4):456-481, 2006b. ISSN 00137944. doi: 10.1016/j.engfracmech.2005.07.012.

D. I. Garagash. Propagation of a plane-strain fluid-driven fracture with a fluid lag: early-time solution. International Journal of Solids and Structures, 43:5811-5835, 2006c.

D. I. Garagash. Scaling of physical processes in fluid-driven fracture: perspective from the tip. In F.M. Borodich, editor, IUTAM Symposium on Scaling in Solid Mechanics, volume 10 of Iutam Bookseries, pages 91-100, Dordrecht, 2009. Springer. ISBN 978-1-4020-9032-5. doi: 10.1007/978-1-4020-9033-2. URL http: //www . springerlink. com/index/10.1007/978-1-4020-9033-2http: //www.springerlink. com/index/j03u4032w300j8w3.pdf.

D. I. Garagash and E. Detournay. The tip region of a fluid-driven fracture in an elastic medium. ASME Journal of Applied Mechanics, 67:183-192, 2000.

D. I. Garagash and E. Detournay. Plane-strain propagation of a fluid-driven fracture: small toughness solution. ASME Journal of Applied Mechanics, 72:916-928, 2005.

D. I. Garagash, E. Detournay, and J. I. Adachi. Multiscale tip asymptotics in hydraulic fracture with leak-off. Journal of Fluid Mechanics, 669:260-297, 2011. doi: 10.1017/S002211201000501X. URL http: //www . journals . cambridge .org/ abstract_S002211201000501X.

J. Geertsma and F. De Klerk. A rapid method of predicting width and extent of hydraulically induced fractures. Journal of Petroleum Technology, 21(12):15711581, 1969.

L. N. Germanovich, R. S. Hurt, J. A. Ayoub, E. Siebrits, D. Norman, I. Ispas, and C. T. Montgomery. Experimental study of hydraulic fracturing in unconsolidated materials. In SPE International Symposium and Exhibition on Formation Damage Control. Society of Petroleum Engineers, 2012. 
S. V. Golovin, V. I. Isaev, A. N. Baykin, D. S. Kuznetsov, and A. E. Mamontov. Hydraulic fracture numerical model free of explicit tip tracking. International Journal of Rock Mechanics and Mining Sciences, 76:174-181, 2015.

E. Gordeliy and E. Detournay. A fixed grid algorithm for simulating the propagation of a shallow hydraulic fracture with a fluid lag. International Journal for Numerical and Analytical Methods in Geomechanics, 35(5):602-629, 2010. ISSN 03639061. doi: 10.1002/nag.913.

E. Gordeliy and A. P. Peirce. Coupling schemes for modeling hydraulic fracture propagation using the xfem. Computer Methods in Applied Mechanics and Engineering, 253:305-322, 2013a.

E. Gordeliy and A. P. Peirce. Implicit level set schemes for modeling hydraulic fractures using the xfem. Computer Methods in Applied Mechanics and Engineering, 266:125-143, 2013b.

E. Gordeliy and A. P. Peirce. Enrichment strategies and convergence properties of the XFEM for hydraulic fracture problems. Computer Methods in Applied Mechanics and Engineering, 283:474-502, 2015.

P. Grassl, C. Fahy, D. Gallipoli, and S. J. Wheeler. On a 2D hydro-mechanical lattice approach for modeling hydraulic fracture. Journal of the Mechanics and Physics of Solids, 75:104-118, 2015.

J. P. Gray and J. J. Monaghan. Numerical modelling of stress fields and fracture around magma chambers. Journal of Volcanology and Geothermal Research, 135 (3):259-283, 2004.

P. Gupta and C. A. Duarte. Simulation of non-planar three-dimensional hydraulic fracture propagation. International Journal for Numerical and Analytical Methods in Geomechanics, 38(13):1397-1430, 2014.

P. Gupta and C. A. Duarte. Coupled formulation and algorithms for the simulation of non-planar three-dimensional hydraulic fractures using the generalized finite element method. International Journal for Numerical and Analytical Methods in Geomechanics, 2015.

W. E. Hassebroek and A. B. Waters. Advancements through 15 years of fracturing. Journal of Petroleum Technology, 16(7):760-764, 1964. 
D. A. Hills, P. A. Kelly, D. N. Dai, and A. M. Korsunsky. Solution of crack problems: the distributed dislocation technique, volume 44 of Solid Mechanics and its Applications. Kluwer Academic Publ., Dordrecht, 1996.

S. Hormozi and I. A. Frigaard. Dispersion of solids in fracturing flows of yield stress fluids. Journal of Fluid Mechanics, 830:93-137, 2017.

G. C. Howard and C. R. Fast. Optimum fluid characteristics for fracture extension. Drilling and Production Practice, pages 261-270, 1957.

J. Hu and D. I. Garagash. Plane-strain propagation of a fluid-driven crack in a permeable rock with fracture toughness. Journal of Engineering Mechanics, 136 (9):1152, 2010. ISSN 07339399. doi: 10.1061/(ASCE)EM.1943-7889.0000169. URL http://link.aip.org/link/JENMDT/v136/i9/p1152/s1\&Agg=doi.

H. Huang and E. Detournay. Intrinsic length scales in tool-rock interaction. International Journal of Geomechanics, 8(1):39-44, 2008.

H. Huang, B. Lecampion, and E. Detournay. Discrete element modeling of tool-rock interaction i: Rock cutting. International Journal for Numerical and Analytical Methods in Geomechanics, 137(13):1913-1929, 2013. doi: 10.1002/nag.2113.

M. J. Hunsweck, Y. Shen, and A. J. Lew. A finite element approach to the simulation of hydraulic fractures with lag. International Journal for Numerical and Analytical Methods in Geomechanics, 37(9):993-1015, 2013.

J. W. Hutchinson and Z. Suo. Mixed mode cracking in layered materials. Advances in Applied Mechanics, 29:63-191, 1991.

R. G. Jeffrey and A. P. Bunger. A detailed comparison of experimental and numerical data on hydraulic fracture height growth through stress contrasts. SPE Journal, 14(3):413-422, 2009.

R. G. Jeffrey, A. P. Bunger, B. Lecampion, X. Zhang, Z. Chen, A. van As, D. Allison, W. De Beer, J. Dudley, E. Siebrits, et al. Measuring hydraulic fracture growth in naturally fractured rock. In SPE Annual Technical Conference and Exhibition, 2009. SPE 124919.

O. C. Jones. An improvement in the calculation of turbulent friction in rectangular ducts. Journal of Fluids Engineering, 98(2):173-180, 1976. 
M. F. Kanninen and C. H. Popelar. Advanced Fracture Mechanics, volume 15 of The Oxford Engineering Science Series. Oxford University Press, Oxford UK, 1985.

B. L. Karihaloo and Q. Z. Xiao. Modelling of stationary and growing cracks in fe framework without remeshing: a state-of-the-art review. Computers \& Structures, 81(3):119-129, 2003.

L. Kemp. Study of Nordgren's equation of hydraulic fracturing. SPE Production Engineering, 1990. SPE-18959.

A. R. Khoei, M. Hirmand, M. Vahab, and M. Bazargan. An enriched FEM technique for modeling hydraulically driven cohesive fracture propagation in impermeable media with frictional natural faults: numerical and experimental investigations. International Journal for Numerical Methods in Engineering, 104(6): 439-468, 2015.

S. Khristianovic and Y. Zheltov. Formation of vertical fractures by means of highly viscous fluids. In Proc., 4th World Petroleum Congress, volume II, pages 579-586, Rome, 1955.

Y. Kovalyshen. Fluid-Driven Fracture in Poroelastic Medium. PhD thesis, University of Minnesota, Minneapolis, MN, 2010.

Y. Kovalyshen and E. Detournay. A reexamination of the classical PKN model of hydraulic fracture. Transport in Porous Media, 81(2):317-339, 2009. ISSN 0169-3913. doi: 10.1007/s11242-009-9403-4.

Y. Kovalyshen and E. Detournay. Fluid-driven fracture in a poroelastic rock. In ISRM International Conference for Effective and Sustainable Hydraulic Fracturing. International Society for Rock Mechanics, 2013.

O. Kresse, X. Weng, D. A. Chuprakov, R. Prioul, C. Cohen, et al. Effect of flow rate and viscosity on complex fracture development in ufm model. In ISRM International Conference for Effective and Sustainable Hydraulic Fracturing. International Society for Rock Mechanics, 2013.

P. Krysl and T. Belytschko. The element free galerkin method for dynamic propagation of arbitrary 3-D cracks. International Journal for Numerical Methods in Engineering, 44(6):767-800, 1999. 
D. Kumar and A. Ghassemi. 3D proroelastic simulation and analysis of multiple fracture propagation and refracturing of closely-spaced horizontal wells. In the 50th US Rock Mechanics/ Geomechanics Symposium, Houston, Texas, 2016.

A. Lakirouhani, E. Detournay, and A. P. Bunger. A reassessment of in situ stress determination by hydraulic fracturing. Geophysical Journal International, 205(3): 1859-1873, 2016.

V. Lazarus. Brittle fracture and fatigue propagation paths of 3D plane cracks under uniform remote tensile loading. International journal of Fracture, 122(1):23-46, 2003.

V. Lazarus. Perturbation approaches of a planar crack in linear elastic fracture mechanics: a review. Journal of the Mechanics and Physics of Solids, 59(2): 121-144, 2011.

J. B. Leblond, V. Lazarus, and A. Karma. Multiscale cohesive zone model for propagation of segmented crack fronts in mode I+III fracture. International Journal of Fracture, 191(1-2):167-189, 2015.

B. Lecampion. An extended finite element method for hydraulic fracture problems. International Journal for Numerical Methods in Biomedical Engineering, 25:121133, 2009. doi: 10.1002/cnm.1111.

B. Lecampion and J. Desroches. Simultaneous initiation and growth of multiple radial hydraulic fractures from a horizontal wellbore. Journal of the Mechanics and Physics of Solids, 82:235-258, 2015a. doi: 10.1016/j.jmps.2015.05.010.

B. Lecampion and J. Desroches. Robustness to formation geological heterogeneities of the limited entry technique for multi-stage fracturing of horizontal wells. Rock Mechanics Rock Engineering, 48(6):2637-2644, 2015b. doi: $10.1007 / \mathrm{s} 00603-015-0836-5$.

B. Lecampion and E. Detournay. An implicit algorithm for the propagation of a hydraulic fracture with a fluid lag. Computer Methods in Applied Mechanics and Engineering, 196:4863-4880, 2007. doi: 10.1016/j.cma.2007.06.011.

B. Lecampion and D. I. Garagash. Confined flow of suspensions modeled by a frictional rheology. Journal of Fluid Mechanics, 759:197-235, 2014. doi: 10.1017/ jfm.2014.557. 
B. Lecampion, A. P. Peirce, E. Detournay, X. Zhang, Z. Chen, A. P. Bunger, C. Detournay, J. Napier, S. Abbas, D. Garagash, and P. Cundall. The impact of the near-tip logic on the accuracy and convergence rate of hydraulic fracture simulators compared to reference solutions. In The International Conference for Effective and Sustainable Hydraulic Fracturing, May 20-22, Brisbane, Australia, 2013.

B. Lecampion, J. Desroches, X. Weng, J. Burghardt, and E. T. Brown. Can we engineer better multistage horizontal completions? evidence of the importance of near-wellbore fracture geometry from theory, lab and field experiments. In $S P E$ Hydraulic Fracturing Technology Conference, 2015. SPE 173363.

B. Lecampion, J. Desroches, R. G. Jeffrey, and A. P. Bunger. Experiments versus theory for the initiation and propagation of radial hydraulic fractures in low permeability materials. Journal of Geophysical Research: Solid Earth, 122, 2017. ISSN 2169-9356. doi: 10.1002/2016JB013183.

S. Lee, M. F. Wheeler, and T. Wick. Pressure and fluid-driven fracture propagation in porous media using an adaptive finite element phase field model. Computer Methods in Applied Mechanics and Engineering, 305:111-132, 2016.

B. Lenoach. The crack tip solution for hydraulic fracturing in a permeable solid. Journal of the Mechanics and Physics of Solids, 43(7):1025-1043, 1995.

T. Lhomme, E. Detournay, and R. G. Jeffrey. Effect of fluid compressibility and borehole radius on the propagation of a fluid-driven fracture. In Proceedings of 11th International Conference on Fracture, Turin, Italy, 2005.

L. D. Libersky and A. G. Petschek. Smooth particle hydrodynamics with strength of materials. In Advances in the free-Lagrange method including contributions on adaptive gridding and the smooth particle hydrodynamics method, pages 248-257. Springer, 1991.

B. Lin, M. E. Mear, and K. Ravi-Chandar. Criterion for initiation of cracks under mixed-mode I+ III loading. International Journal of Fracture, 165(2):175-188, 2010 .

M. Madyarova. Fluid-driven penny-shaped fracture in permeable rock. Master's thesis, University of Minnesota, Minneapolis, MN, USA, 2003.

P. Manneville. Transition to turbulence in wall-bounded flows: Where do we stand? Mechanical Engineering Reviews, (0), 2016. 
M. W. McClure and R. N. Horne. Discrete Fracture Network Modeling of Hydraulic Stimulation: Coupling Flow and Geomechanics. Springer Briefs in Earth Sciences. Springer, New York, 2013.

J. M. Melenk and I. Babuška. The partition of unity finite element method: basic theory and applications. Computer Methods in Applied Mechanics and Engineering, 139(1-4):289-314, 1996.

D. A. Mendelsohn. A review of hydraulic fracture modeling - part I: general concepts, 2D models, motivation for 3D modeling. Journal of Energy Resources Technology, 106(3):369-376, 1984a.

D. A. Mendelsohn. A review of hydraulic fracture modeling-II: 3D modeling and vertical growth in layered rock. Journal of Energy Resources Technology, 106(4): 543-553, 1984b.

G. Meschke and D. Leonhart. A generalized finite element method for hydromechanically coupled analysis of hydraulic fracturing problems using space-time variant enrichment functions. Computer Methods in Applied Mechanics and Engineering, 290:438-465, 2015.

B. R. Meyer. Three-dimensional hydraulic fracturing simulation on personal computers: theory and comparison studies. In Proceedings SPE Eastern Regional Meeting, Morgantown, West Virginia, 24-27 October 1989. SPE 19329.

B. R. Meyer and L. Bazan. A discrete fracture network model for hydraulically induced fractures-theory, parametric and case studies. In Proceedings SPE Hydraulic Fracturing Technology Conference and Exhibition, The Woodlands, Texas, USA, 2011. SPE 140514.

C. Miehe and S. Mauthe. Phase field modeling of fracture in multi-physics problems. part iii. crack driving forces in hydro-poro-elasticity and hydraulic fracturing of fluid-saturated porous media. Computer Methods in Applied Mechanics and Engineering, 304:619-655, 2016.

C. Miehe, F. Welschinger, and M. Hofacker. Thermodynamically consistent phasefield models of fracture: variational principles and multi-field fe implementations. International Journal for Numerical Methods in Engineering, 83(10):1273-1311, 2010. 
C. Miehe, S. Mauthe, and S. Teichtmeister. Minimization principles for the coupled problem of Darcy-Biot-type fluid transport in porous media linked to phase field modeling of fracture. Journal of the Mechanics and Physics of Solids, 82:186-217, 2015.

A. Mikelic, M. F. Wheeler, and T. Wick. A phase-field method for propagating fluidfilled fractures coupled to a surrounding porous medium. Multiscale Modeling $\mathfrak{6}$ Simulation, 13(1):367-398, 2015.

N. Moës, J. Dolbow, and T. Belytcshko. A finite element method for crack growth without remeshing. International Journal for Numerical Methods in Engineering, 46:131-150, 1999.

S. G. Mogilevskaya. Lost in translation: crack problems in different languages. International Journal of Solids and Structures, 51(25):4492-4503, 2014.

T. Mohammadnejad and J. E. Andrade. Numerical modeling of hydraulic fracture propagation, closure and reopening using XFEM with application to in-situ stress estimation. International Journal for Numerical and Analytical Methods in Geomechanics, 40(15):2033-2060, 2016. doi: 10.1002/nag.2512.

T. Mohammadnejad and A. R. Khoei. An extended finite element method for hydraulic fracture propagation in deformable porous media with the cohesive crack model. Finite Elements in Analysis and Design, 73:77-95, 2013a.

T. Mohammadnejad and A. R. Khoei. Hydro-mechanical modeling of cohesive crack propagation in multiphase porous media using the extended finite element method. International Journal for Numerical and Analytical Methods in Geomechanics, 37 (10):1247-1279, 2013b. doi: 10.1002/nag.2079.

J. J. Monaghan. Smoothed particle hydrodynamics. Annual Review of Astronomy and Astrophysics, 30(1):543-574, 1992.

J. J. Monaghan. Smoothed particle hydrodynamics. Reports on Progress in Physics, 68(8):1703, 2005.

C. T. Montgomery and M. B. Smith. Hydraulic fracturing: history of an enduring technology. Journal of Petroleum Technology, 62(12):26-40, 2010. 
N. Nagel, I. Gil, M. Sanchez-Nagel, and B. Damjanac. Simulating hydraulic fracturing in real fractured rocks - overcoming the limits of pseudo3D models. In Proceedings SPE Hydraulic Fracturing Technology Conference and Exhibition, The Woodlands, Texas, USA, January 24-26 2011. SPE 140480.

J. A. L. Napier and E. Detournay. Propagation of non-planar pressurized cracks from a borehole. In the 5th International Conference on Structural Engineering, Mechanics and Computation, pages 597-602, 2013.

A. Needleman. Some issues in cohesive surface modeling. Procedia IUTAM, 10: 221-246, 2014.

J. Nikuradse. Laws of flow in rough pipes. National Advisory Committee for Aeronautics Washington, 1950. Translation of the 1933 paper.

K. G. Nolte. Determination of fracture parameters from fracturing pressure decline. In Proc. SPE Annual Technical Conference and Exhibition, Las Vegas, 1979. SPE 8341.

K. G. Nolte. Determination of proppant and fluid schedules from fracturing-pressure decline. SPE Production Engineering, 1(4):255-265, 1986. (SPE 13278).

K. G. Nolte and M. B. Smith. Interpretation of fracturing pressures. Journal of Petroleum Technology, 33(9):1767-1775, 1981.

R. P. Nordgren. Propagation of vertical hydraulic fractures. Society of Petroleum Engineers Journal, 253:306-314, 1972.

J. E. Olson and A. Dahi-Taleghani. Modeling simultaneous growth of multiple hydraulic fractures and their interaction with natural fractures. In Proceedings SPE Hydraulic Fracturing Technology Conference and Exhibition, The Woodlands, Texas, USA, January 19-21 2009. SPE 119739.

A. A. Osiptsov. Fluid mechanics of hydraulic fracturing: a review. Journal of Petroleum Science and Engineering, 2017.

H. Ouchi, A. Katiyar, J. York, J. T. Foster, and M. M. Sharma. A fully coupled porous flow and geomechanics model for fluid driven cracks: a peridynamics approach. Computational Mechanics, 55(3):561-576, 2015. 
A. Paluszny and R. W. Zimmerman. Numerical simulation of multiple 3D fracture propagation using arbitrary meshes. Computer Methods in Applied Mechanics and Engineering, 200(9):953-966, 2011.

E. Papachristos, L. Scholtès, F. V. Donzé, and B. Chareyre. Intensity and volumetric characterizations of hydraulically driven fractures by hydro-mechanical simulations. International Journal of Rock Mechanics and Mining Sciences, 93: 163-178, 2017.

P. Papanastasiou. The influence of plasticity in hydraulic fracturing. International Journal of Fracture, 84(1):61-79, 1997.

P. Papanastasiou and M. Thiercelin. Influence of inelastic rock behaviour in hydraulic fracturing. In International Journal of Rock Mechanics and Mining Sciences $\&$ Geomechanics Abstracts, volume 30, pages 1241-1247. Elsevier, 1993.

K. Park and G. H. Paulino. Cohesive zone models: a critical review of tractionseparation relationships across fracture surfaces. Applied Mechanics Reviews, 64 (6):060802, 2011.

A. P. Peirce. Localized jacobian ILU preconditioners for hydraulic fractures. International Journal for Numerical Methods in Engineering, 2005. in press.

A. P. Peirce. Modeling multi-scale processes in hydraulic fracture propagation using the implicit level set algorithm. Computer Methods in Applied Mechanics and Engineering, 283:881-908, 2015.

A. P. Peirce. Implicit level set algorithms for modelling hydraulic fracture propagation. Philosophical Transactions of the Royal Society of London A: Mathematical, Physical and Engineering Sciences, 374(2078):20150423, 2016.

A. P. Peirce and E. Detournay. An implicit level set method for modeling hydraulically driven fractures. Computer Methods in Applied Mechanics and Engineering, 197(33-40):2858-2885, 2008. ISSN 00457825. doi: 10.1016/j.cma.2008.01.013. URL http://linkinghub.elsevier.com/retrieve/pii/S0045782508000443.

A. P. Peirce and E. Detournay. An Eulerian moving front algorithm with weak-form tip asymptotics for modeling hydraulically driven fractures. Communications in Numerical Methods in Engineering, 25(2):185-200, 2009. ISSN 1099-0887. doi: 10. 1002/cnm. URL http://onlinelibrary.wiley.com/doi/10.1002/cnm.1119/ abstract. 
A. P. Peirce and E. Siebrits. A dual mesh multigrid preconditioner for the efficient solution of hydraulically driven fracture problems. International Journal for Numerical Methods in Engineering, 63:1797-1823, 2005.

T. K. Perkins and L. R. Kern. Widths of hydraulic fractures. Journal of Petroleum Technology, 222:937-949, 1961.

K. H. Pham and K. Ravi-Chandar. On the growth of cracks under mixed-mode I+ III loading. International Journal of Fracture, 199(1):105-134, 2016.

M. Profit, M. Dutko, J. Yu, S. Cole, D. Angus, and A. Baird. Complementary hydro-mechanical coupled finite/discrete element and microseismic modelling to predict hydraulic fracture propagation in tight shale reservoirs. Computational Particle Mechanics, 3(2):229-248, 2016a.

M. L. Profit, M. Dutko, and J. Yu. Developing a framework to simulate the hydraulic fracturing of tight gas reservoirs based on integrative adaptive remeshing and combined finite/discrete element approach. In 49th US Rock Mechanics/Geomechanics Symposium, number ARMA-2015-293. American Rock Mechanics Association, 2015.

M. L. Profit, M. Dutko, J. Yu, J. Armstrong, D. Parfitt, and U. Mutlu. Application of state of the art hydraulic fracture modelling techniques for safe-optimized design and for enhanced production. In 50th US Rock Mechanics/Geomechanics Symposium, number ARMA-2016-792. American Rock Mechanics Association, 2016b.

A. Quarteroni, R. Sacco, and F. Saleri. Numerical Mathematics. Texts in applied mathematics. Springer, 2000.

J. R. Rice. Mathematical analysis in the mechanics of fracture. In H. Liebowitz, editor, Fracture: An Advanced Treatise, volume 2, chapter 3, pages 191-311. 1968.

J. R. Rice and D. C. Drucker. Energy changes in stressed bodies due to void and crack growth. International Journal of Fracture Mechanics, 3:19-27, 1967.

J. Rungamornrat, M. F. Wheeler, and M. E. Mear. A numerical technique for simulating nonplanar evolution of hydraulic fractures. In the 2005 Annual Technical Conference and Exhibition, Dallas, Texas, 9-12 October 20052005.

S. Salimzadeh and N. Khalili. A three-phase xfem model for hydraulic fracturing with cohesive crack propagation. Computers and Geotechnics, 69:82-92, 2015. 
S. Salimzadeh, A. Paluszny, and R. W. Zimmerman. Three-dimensional poroelastic effects during hydraulic fracturing in permeable rocks. International Journal of Solids and Structures, 2016.

S. Samimi and A. Pak. A fully coupled element-free galerkin model for hydromechanical analysis of advancement of fluid-driven fractures in porous media. International Journal for Numerical and Analytical Methods in Geomechanics, 40:2178-2206, 2016.

D. Santillán, R. Juanes, and L. Cueto-Felgueroso. Phase field model of fluid-driven fracture in elastic media: Immersed-fracture formulation and validation with analytical solutions. Journal of Geophysical Research: Solid Earth, 122(4):2565-2589, 2017.

E. Sarris and P. Papanastasiou. Modelling of hydraulic fracturing in a poroelastic cohesive formation. International Journal of Geomechanics, 2011. ISSN 15323641. doi: 10.1061/(ASCE)GM.1943-5622.0000121.

E. Sarris and P. Papanastasiou. Numerical modeling of fluid-driven fractures in cohesive poroelastoplastic continuum. International Journal for Numerical and Analytical Methods in Geomechanics, 37(12):1822-1846, 2013.

E. Sarvaramini and D. I. Garagash. Breakdown of a pressurized fingerlike crack in a permeable solid. Journal of Applied mechanics, 82:061006-1-061006-10, 2015.

A. Savitski and E. Detournay. Propagation of a penny-shaped fluid-driven fracture in an impermeable rock: asymptotic solutions. International Journal of Solids and Structures, 39(26):6311-6337, dec 2002. ISSN 00207683. doi: 10.1016/S0020-7683(02)00492-4.

S. Secchi, L. Simoni, and B. A. Schrefler. Mesh adaptation and transfer schemes for discrete fracture propagation in porous materials. International Journal for Numerical and Analytical Methods in Geomechanics, 31(2):331-345, 2007. doi: $10.1002 /$ nag. 581 .

R. R. Settgast, P. Fu, S. D. C. Walsh, J. A. White, C. Annavarapu, and F. J. Ryerson. A fully coupled method for massively parallel simulation of hydraulically driven fractures in 3-dimensions. International Journal for Numerical and Analytical Methods in Geomechanics, 2016. 
E. Siebrits and A. P. Peirce. An efficient multi-layer planar 3D fracture growth algorithm using a fixed mesh approach. International Journal for Numerical Methods in Engineering, 53:691-717, 2002.

S. A. Silling. Reformulation of elasticity theory for discontinuities and long-range forces. Journal of the Mechanics and Physics of Solids, 48:175-209, 2000.

L. Simoni and S. Secchi. Cohesive fracture mechanics for a multi-phase porous medium. Engineering Computations, 20(5/6):675-698, 2003. ISSN 0264-4401. doi: 10.1108/02644400310488817. URL http://www.emeraldinsight.com/10. $1108 / 02644400310488817$.

E. R. Simonson, A. S. Abou-Sayed, and R. J. Clifton. Containment of massive hydraulic fractures. SPE Journal, 18(1):27-32, 1978.

M. B. Smith and C. T. Montgomery. Hydraulic Fracturing. Crc Press, 2015.

D.A. Spence and P. Sharp. Self-similar solutions for elastohydrodynamic cavity flow. Proceedings of the Royal Society of London. A. Mathematical and Physical Sciences, 400(1819):289-313, 1985.

A. M. Starfield and P. A. Cundall. Towards a methodology for rock mechanics modelling. In International Journal of Rock Mechanics and Mining Sciences 6 Geomechanics Abstracts, volume 25, pages 99-106, 1988.

N. Sukumar, B. Moran, T. Black, and T. Belytschko. An element-free galerkin method for three-dimensional fracture mechanics. Computational Mechanics, 20 (1):170-175, 1997.

A. Z. Szeri. Fluid Film Lubrication. Cambridge University Press, 2010.

V. Tvergaard and J. W. Hutchinson. The relation between crack growth resistance and fracture process parameters in elastic-plastic solids. Journal of the Mechanics and Physics of Solids, 40:1377-1397, 1992.

L. Vandamme and J. H. Curran. A three-dimensional hydraulic fracturing simulator. International Journal for Numerical Methods in Engineering, 28:909-927, 1989.

P. S. Virk. Drag reduction fundamentals. AIChE Journal, 21(4):625-656, 1975. 
N. R. Warpinski and L. W. Teufel. Influence of geologic discontinuities on hydraulic fracture propagation (includes associated papers 17011 and 17074). Journal of Petroleum Technology, 39(02):209-220, 1987.

N. R. Warpinski, I. S. Abou-Sayed, Z. Moschovidis, and C. Parker. Hydraulic fracture model comparison study: complete results. Technical Report SAND93-7042, Sandia National Laboratories, 1993.

N. R. Warpinski, Z. A. Moschovidis, C. D. Parker, and I. S. Abou-Sayed. Comparison study of hydraulic fracturing models - test case: GRI staged field experiment No. 3 (includes associated paper 28158). SPE Production \&3 Facilities, 9(01 (SPE25890)):7-16, 1994.

X. Weng. Incorporation of 2D fluid flow into a pseudo-3D hydraulic fracturing simulator. SPE Production Engineering, November:331-337, 1992.

X. Weng, O. Kresse, C. E. Cohen, R. Wu, and H. Gu. Modeling of hydraulicfracture-network propagation in a naturally fractured formation. SPE Production Es Operations, 26(04):368-380, 2011a.

X. Weng, O. Kresse, C. E. Cohen, R. Wu, and H. Gu. Modeling of hydraulic fracture network propagation in a naturally fractured formation. In Proceedings SPE Hydraulic Fracturing Technology Conference and Exhibition, The Woodlands, Texas, USA, 2011b. SPE 140253.

Z. A. Wilson and C. M. Landis. Phase-field modeling of hydraulic fracture. Journal of the Mechanics and Physics of Solids, 96:264-290, 2016.

P. A. Witherspoon. Validity of cubic law for fluid flow in a deformable rock fracture. Water Resources Res., 16(6):1016-1024, 1980.

M. Wrobel and G. Mishuris. Efficient pseudo-spectral solvers for the pkn model of hydrofracturing. International Journal of Fracture, 184(1-2):151-170, 2013.

Michal Wrobel and Gennady Mishuris. Hydraulic fracture revisited: Particle velocity based simulation. International Journal of Engineering Science, 94:23-58, 2015.

K. Wu and J. E. Olson. Simultaneous multifracture treatments: fully coupled fluid flow and fracture mechanics for horizontal wells. SPE Journal, April 2015a. doi: http://dx.doi.org/10.2118/167626-PA. SPE-167626-PA. 
K. Wu and J. E. Olson. A simplified three-dimensional displacement discontinuity method for multiple fracture simulations. International Journal of Fracture, 193 (2):191-204, 2015b.

R. Wu, A. P. Bunger, R. G. Jeffrey, and E. Siebrits. A comparison of numerical and experimental results of hydraulic fracture growth into a zone of lower confining stress. In The 42nd US Rock Mechanics Symposium (USRMS). American Rock Mechanics Association, 2008.

P. Xing, K. Yoshioka, J. I. Adachi, A. El-Fayoumi, and A. P. Bunger. Laboratory measurement of tip and global behavior for zero-toughness hydraulic fractures with circular and blade-shaped (pkn) geometry. Journal of the Mechanics and Physics of Solids, 104:172-186, 2017.

P. Xing, K. Yoshioka, J. I. Adachi, A. El-Fayoumi, and A. P. Bunger. Laboratory demonstration of hydraulic fracture height growth across weak discontinuities. Geophysics, In Review.

G. Xu and S. W. Wong. Interaction of multiple non-planar hydraulic fractures in horizontal wells. In IPTC 2013: International Petroleum Technology Conference, Beijing, China, 26-28 March 2013. IPTC 17043-MS.

K. Yamamoto, T. Shimamoto, and S. Sukemura. Multiple fracture propagation model for a three-dimensional hydraulic fracturing simulator. International Journal of Geomechanics, 4(1):46-57, 2004.

C. Yan and H. Zheng. Three-dimensional hydromechanical model of hydraulic fracturing with arbitrarily discrete fracture networks using finite-discrete element method. International Journal of Geomechanics, page 04016133, 2016.

Y. Yao, L. Liu, and L. M. Keer. Pore pressure cohesive zone modeling of hydraulic fracture in quasi-brittle rocks. Mechanics of Materials, 83:17-29, 2015.

F. Zhang, E. V. Dontsov, and M. Mack. Fully coupled simulation of a hydraulic fracture interacting with natural fractures with a hybrid discrete-continuum method. International Journal for Numerical and Analytical Methods in Geomechanics, 2017a.

X. Zhang and R. G. Jeffrey. Role of overpressurized fluid and fluid-driven fractures in forming fracture networks. Journal of Geochemical Exploration, 144:194-207, 2014. 
X. Zhang, R. G. Jeffrey, and M. Thiercelin. Deflection and propagation of fluiddriven fractures at frictional bedding interfaces: A numerical investigation. Journal of Structural Geology, 30:396-410, 2007.

X. Zhang, B. Wu, R. G. Jeffrey, L. D. Connell, and G. Zhang. A pseudo-3D model for hydraulic fracture growth in a layered rock. International Journal of Solids and Structures, 115:208-223, 2017b.

H. Zia and B. Lecampion. Propagation of a height contained hydraulic fracture in turbulent flow regimes. International Journal of Solids and Structures, 110-111: 265-278, April 2017. doi: 10.1016/j.ijsolstr.2016.12.029. 